\title{
Closed Hypersurfaces Driven by Mean Curvature and Inner Pressure
}

\author{
THILO NOTZ \\ Max Planck Institute for Gravitational Physics (Albert Einstein Institute)
}

\begin{abstract}
We introduce a hyperbolic equation that describes the motion of closed hypersurfaces in a Riemannian manifold with surface tension and inner pressure as driving forces. In the case of spherical surfaces this equation can be considered as an idealized mathematical model for a moving soap bubble. The equation is derived as an Euler-Lagrange equation from a suitable action integral. It is a quasi-linear degenerate hyperbolic PDE of second order that describes the motion of the surfaces extrinsically.

Our main results are the solution of the Cauchy problem by means of the Nash-Moser inverse function theorem, a continuation criterion, and stability estimates. (C) 2012 Wiley Periodicals, Inc.
\end{abstract}

\section{Introduction}

Let $\mathcal{N}$ be a smooth, closed, oriented manifold of dimension $n$, and let $\left(\mathcal{M}^{n+1}, \bar{g}\right)$ be a smooth, complete, oriented, $n+1$-dimensional Riemannian manifold. We want to derive an equation of motion for closed hypersurfaces with surface tension and inner pressure as driving forces. If $\mathcal{N}$ is the $n$-sphere, this equation can be considered as an idealized model for a moving soap bubble. For a smooth family of immersions $u:[0, T] \times \mathcal{N} \rightarrow \mathcal{M}$ we define an action integral of the form

$$
\mathcal{A}(u)=\int_{0}^{T} \mathcal{K}(u)-\mathcal{J}(u)-\mathcal{J}(u) d t
$$

where $\mathcal{K}$ is the kinetic energy and $\mathcal{J}, \mathcal{J}$ contribute to the potential energy. We choose $\mathcal{J}(u)$ as the energy of the surface tension, i.e., the surface area

$$
\mathcal{J}(u)=\int_{\mathcal{N}} d \mu_{t}
$$

Here $d \mu_{t}$ denotes the induced surface measure of the induced metric $g(t)=$ $u(t)^{*} \bar{g}$ at time $t$.

The inner pressure is motivated by that of an ideal gas with constant temperature; i.e., it is proportional to $\operatorname{Vol}(u)^{-1}$ where $\operatorname{Vol}(u)$ is the enclosed volume of the 
surface $u(\mathcal{N})$. Therefore we define for a parameter $\varrho>0$

$$
\mathcal{J}(u)=-\varrho \log \left(\frac{\operatorname{Vol}(u)}{\operatorname{Vol}_{0}}\right) .
$$

The constant $\varrho$ as well as the initial enclosed volume $\mathrm{Vol}_{0}$ are included for scaling reasons. Of course other functions of the enclosed volume could be considered if they lead to a lower volume bound as in Corollary 2.2 below.

In order to define the kinetic energy we fix a reference measure $d \hat{\mu}$ on $\mathcal{N}$ with a smooth density function defining a mass distribution on $\mathcal{N}$. We then integrate the kinetic energies $\frac{1}{2}\left|\partial_{t} u\right|^{2} d \hat{\mu}$ of all the points of the surface and define

$$
\mathcal{K}(u)=\int_{\mathcal{N}} \frac{1}{2}\left|\partial_{t} u\right|^{2} d \hat{\mu} .
$$

This then describes the physical energy of the point particles making up the surface. Altogether the action integral is

$$
\mathcal{A}(u)=\int_{0}^{T} \int_{\mathcal{N}} \frac{1}{2}\left|\partial_{t} u\right|^{2} d \hat{\mu} d t-\int_{0}^{T} \int_{\mathcal{N}} d \mu_{t} d t+\varrho \int_{0}^{T} \log \left(\frac{\operatorname{Vol}(u)}{\operatorname{Vol}_{0}}\right) d t .
$$

The equation we study in this paper is the Euler-Lagrange equation of $\mathcal{A}$. It is readily obtained as

$$
\bar{\nabla}_{\partial_{t}} \partial_{t} u=\frac{d \mu_{t}}{d \widehat{\mu}}\left(-H(u)+\frac{\varrho}{\operatorname{Vol}(u)}\right) v
$$

where $H(u)$ denotes the mean curvature of $u(\mathcal{N})$ with respect to the outer unit normal $v$. By $\bar{\nabla}_{\partial_{t}}$ we denote the covariant derivative along $u$, i.e.,

$$
\bar{\nabla}_{\partial_{t}} \partial_{t} u^{\alpha}=\partial_{t}^{2} u^{\alpha}+\bar{\Gamma}_{\beta \gamma}^{\alpha}(u) \partial_{t} u^{\beta} \partial_{t} u^{\gamma}
$$

with $\bar{\Gamma}_{\beta \gamma}^{\alpha}$ being the Christoffel symbols of $\bar{g}$. We use the Einstein summation convention; i.e., we sum over repeated upper and lower indices. By $h_{i j}$ we will denote the second fundamental form.

The structure of this equation (EQ) generates interest from a mathematical point of view. Although Einstein's equations have a similar structure they describe the evolution of the geometry via intrinsic quantities. In contrast to wave maps our equation is not semilinear, but rather quasi-linear and degenerate.

One of the few mathematically rigorous studies of equations in this category is the paper of LeFloch and Smozcyk [7]. Some fundamental differences to our equation are noted in Remark 1.1

The outline of this paper is as follows: In Section 2 we derive conservation laws and find special solutions of the equation (EQ) such as oscillating and translating spheres. In Section 3 we define a special kind of linear PDE system that will arise in the linearization of (EQ). We derive estimates for these systems that will allow us to solve the Cauchy problem associated to $(\mathrm{EQ})$ in Section 4 by means of the Nash-Moser inverse function theorem (Theorem 4.1). 
In Section 5 we prove a sufficient condition under which the solution can be extended to a larger time interval (Theorem 5.1). The condition is that the family of parametrizations of the surface and its time derivative are bounded in the spatial $C^{4}$-norm.

In the last section, Section 6, we prove that the distance between two solutions grows at most exponentially fast if they are close to each other initially (Theorem 6.1). This estimate implies the uniqueness of solutions and a lifetime estimate. A similar stability estimate holds if the metric of the ambient manifold is close to the euclidean metric (Theorem 6.4).

The results in this paper are contained with more detailed proofs in the author's thesis [8].

Remark 1.1. In [7] LeFloch and Smozcyk consider an Euler-Lagrange equation coming from an action functional including only kinetic energy and surface tension where they use the induced surface measure to define the kinetic energy. There are some fundamental differences that are due to this different definition of kinetic energy. Most importantly, the equation of LeFloch and Smozcyk is only hyperbolic under certain conditions on the tangential velocity. In contrast to their geometric definition of kinetic energy, we use the more physical definition where the surface has a fixed mass density. As a consequence, adding a constant velocity translation to a solution of our equation yields again a solution (see Section 2.3). They also get a more general conservation of interior momentum which implies that the velocity stays orthogonal if it does so initially. They use this to give a short time existence proof in the case of normal velocity. We remark that this case is a restriction and completely different behavior can occur if one allows tangential velocity. For example, the equation of LeFloch and Smozcyk admits a circle rotating with constant velocity as a solution and also a circle shrinking without tangential velocity to a point in finite time.

\section{Conservation Laws and Special Solutions}

\subsection{Energy Conservation}

Define the energy

$$
\mathcal{E}(u(t, \cdot))=\int_{\mathcal{N}} \frac{1}{2}\left|\partial_{t} u\right|^{2} d \hat{\mu}+\int_{\mathcal{N}} d \mu_{t}-\varrho \log \left(\frac{\operatorname{Vol}(u)}{\operatorname{Vol}_{0}}\right) .
$$

Let $u:[0, T) \times \mathcal{N} \rightarrow \mathcal{M}$ solve $(\mathrm{EQ})$ with $\mathcal{E}_{0}=\mathcal{E}(u(0, \cdot))$.

Proposition 2.1. We have $\mathcal{E}(u(t, \cdot))=\mathcal{E}_{0}$ for all $t \in[0, T)$, and it holds that

$$
\partial_{t}\left(\frac{1}{2}\left|\partial_{t} u\right|^{2}+\frac{d \mu_{t}}{d \hat{\mu}}\right)=\operatorname{div}\left(\partial_{t} u^{\top}\right) \frac{d \mu_{t}}{d \hat{\mu}}+\frac{\varrho}{\operatorname{Vol}(u)}\left\langle\partial_{t} u, v\right\rangle \frac{d \mu_{t}}{d \hat{\mu}}
$$

where $\partial_{t} u^{\top}$ is the tangential part of $\partial_{t} u$. 
The proof is a simple computation and is omitted. From the energy conservation we immediately get very general bounds.

COROLlary 2.2 .

(1) The enclosed volume is bounded from below by

$$
\operatorname{Vol}(u) \geq \operatorname{Vol}_{0} e^{-\frac{\varepsilon_{0}}{\varrho}} .
$$

(2) Assume that an isoperimetric inequality holds on $\mathcal{M}$, namely, that there is a constant $c_{\text {iso }}>0$ such that

$$
\int_{\mathcal{N}} d \mu_{t} \geq c_{\text {iso }} \operatorname{Vol}(u)^{\frac{n}{n+1}} .
$$

Then there is a constant $K$ depending only on $c_{\mathrm{iso}}, \varrho, \mathcal{E}_{0}$, and $\mathrm{Vol}_{0}$ such that $\operatorname{Vol}(u) \leq K$ and consequently

$$
\int_{\mathcal{N}} \frac{1}{2}\left|\partial_{t} u\right|^{2} d \hat{\mu}+\int_{\mathcal{N}} d \mu_{t} \leq \varepsilon_{0}+\varrho \log \left(\frac{K}{\operatorname{Vol}_{0}}\right) .
$$

\subsection{Momentum Conservation}

Let $X$ be a Killing vector field on $\mathcal{M}$. Define the momentum with respect to $X$ of a solution $u$ of (EQ) by

$$
\mathcal{P}_{X}(u(t, \cdot))=\int_{\mathcal{N}}\left\langle\partial_{t} u, X(u)\right\rangle d \hat{\mu} .
$$

Proposition 2.3. Let $u:[0, T) \times \mathcal{N} \rightarrow \mathcal{M}$ solve $(\mathrm{EQ})$. Then $\mathcal{P}_{X}(u(t, \cdot))$ is constant as a function of $t$ and it holds that

$$
\partial_{t}\left\langle\partial_{t} u, X(u)\right\rangle=\operatorname{div} X^{\top} \frac{d \mu_{t}}{d \hat{\mu}}+\frac{\varrho}{\operatorname{Vol}(u)}\langle v, X\rangle \frac{d \mu_{t}}{d \hat{\mu}} .
$$

Proof. Let $\phi_{s}$ be the local flow of $X$, which is by definition an isometry, and put $u_{s}=\phi_{s} \circ u$. We have

$$
\begin{aligned}
\partial_{t}\left\langle\partial_{t} u, X(u)\right\rangle & =-\langle H v, X(u)\rangle \frac{d \mu_{t}}{d \hat{\mu}}+\frac{\varrho}{\operatorname{Vol}(u)}\langle v, X\rangle \frac{d \mu_{t}}{d \hat{\mu}}+\underbrace{\left\langle\partial_{t} u, \bar{\nabla}_{\partial_{t}} X(u)\right\rangle}_{=0} \\
& =\operatorname{div} X^{\top} \frac{d \mu_{t}}{d \hat{\mu}}-\left.\frac{\partial}{\partial s}\right|_{s=0} \log \left(d \mu_{t}\left(u_{s}\right)\right) \frac{d \mu_{t}}{d \hat{\mu}}+\frac{\varrho}{\operatorname{Vol}(u)}\langle v, X\rangle \frac{d \mu_{t}}{d \hat{\mu}} .
\end{aligned}
$$

Now $\left.\frac{\partial}{\partial s}\right|_{s=0} d \mu_{t}\left(u_{s}\right)=0$ as $X$ is Killing. Integrating with respect to $d \hat{\mu}$ and $d t$ and using that $\int_{\mathcal{N}}\langle v, X\rangle d \mu_{t}=\left.\frac{d}{d s}\right|_{s=0} \operatorname{Vol}\left(u_{s}\right)=0$, we get the result.

We can obtain a third conservation law by exploiting another symmetry of the action, namely, the invariance under diffeomorphisms of $\mathcal{N}$ that leave $d \widehat{\mu}$ invariant. So let $Y$ be a vector field on $\mathcal{N}$ with $\operatorname{div}_{d \widehat{\mu}} Y=0$. We define the interior 
momentum with respect to $Y$ as

$$
Q_{Y}(u(t, \cdot))=\int_{\mathcal{N}}\left\langle\partial_{t} u, u_{*} Y\right\rangle d \hat{\mu} .
$$

Proposition 2.4. Let $u:[0, T) \times \mathcal{N} \rightarrow \mathcal{M}$ solve (EQ). Then $Q_{Y}(u(t, \cdot))$ is constant as a function of $t$. Furthermore, we have

$$
\partial_{t}\left\langle\partial_{t} u, u_{*} Y\right\rangle=\frac{1}{2} \operatorname{div}_{d \widehat{\mu}}\left(\left|\partial_{t} u\right|^{2} Y\right)
$$

Proof. In local coordinates on $\mathcal{N}$ write $Y=Y^{i} \partial_{i}$. We have that $u_{*} Y=Y^{i} \partial_{i} u$ and compute

$$
\begin{aligned}
\partial_{t}\left\langle\partial_{t} u, u_{*} Y\right\rangle & =\underbrace{\left\langle\bar{\nabla}_{\partial_{t}} \partial_{t} u, u_{*} Y\right\rangle}_{=0}+\left\langle\partial_{t} u, \bar{\nabla}_{\partial_{t}} \partial_{i} u\right\rangle Y^{i} \\
& =Y^{i} \frac{1}{2} \partial_{i}\left|\partial_{t} u\right|^{2}=\frac{1}{2} \operatorname{div}_{d \hat{\mu}}\left(\left|\partial_{t} u\right|^{2} Y\right)-\frac{1}{2}\left|\partial_{t} u\right|^{2} \operatorname{div}_{d \hat{\mu}} Y .
\end{aligned}
$$

Integrating with respect to $d \widehat{\mu}$ and $d t$ using the divergence theorem and $\operatorname{div}_{d} \widehat{\mu} Y=$ 0 yields the result.

\subsection{Special Solutions}

Assume $u: \mathbb{R} \times \mathbb{S}^{n} \rightarrow \mathbb{R}^{n+1}$ has the form $u(t, x)=r(t) x$ with initial conditions $r(0)=r_{0}>0$ and $\dot{r}(0)=r_{1}$. Let $d \hat{\mu}$ be the surface measure of a spherical metric $g_{0}$, i.e., $g_{0}=\gamma_{0}^{2} g_{\mathbb{S}^{n}}$ where $g_{\mathbb{S}^{n}}$ is the standard metric on $\mathbb{S}^{n}$ and $\gamma_{0}>0$ is a constant. Let $\omega_{n+1}$ denote the volume of the unit ball in $\mathbb{R}^{n+1}$. Equation (EQ) then becomes an ODE for the radius $r(t)$

$$
\ddot{r}(t)=-\frac{n r(t)^{n-1}}{\gamma_{0}^{n}}+\frac{\varrho}{\omega_{n+1} \gamma_{0}^{n} r(t)} .
$$

This second-order ODE can be written as a system of first-order ODEs for $(r, z)=$ $(r, \dot{r})$

$$
\begin{aligned}
& \dot{r}=z, \\
& \dot{z}=-\frac{n r(t)^{n-1}}{\gamma_{0}^{n}}+\frac{\varrho}{\omega_{n+1} \gamma_{0}^{n} r(t)} .
\end{aligned}
$$

Clearly the right-hand side is locally Lipschitz and in fact smooth around $\left(r_{0}, r_{1}\right)$, so there exists a local smooth solution. Using the energy conservation, we can write the integral curves as a graph

$$
z= \pm \sqrt{\frac{2 \varepsilon_{0}}{(n+1) \omega_{n+1} \gamma_{0}^{n}}-2 \frac{r^{n}}{\gamma_{0}^{n}}+\frac{2 \varrho}{\omega_{n+1} \gamma_{0}^{n}} \log \left(\frac{r}{r_{0}}\right)} .
$$

It is easy to check that these curves are closed. We have an equilibrium if initially $r^{n}=\frac{\varrho}{n \omega_{n+1}}$ and $\dot{r}=0$. We summarize this as a proposition. 
Proposition 2.5. Let $g_{0}=\gamma_{0}^{2} g_{\mathbb{S}^{n}}$ be a spherical metric with $\gamma_{0}>0$ and $d \hat{\mu}$ its surface measure. Let $r_{0}>0, r_{1} \in \mathbb{R}$. Then there exists a unique rotationally symmetric periodic solution $u: \mathbb{R} \times \mathbb{S}^{n} \rightarrow \mathbb{R}^{n+1}$ of equation (EQ) centered at the origin with initial conditions $u(0, x)=r_{0} x, \partial_{t} u(0, x)=r_{1} x$. If

$$
r_{0}=\sqrt[n]{\frac{\varrho}{n \omega_{n+1}}} \text { and } r_{1}=0
$$

then the solution is constant in $t$.

If $u:[0, T) \times \mathcal{N} \rightarrow \mathbb{R}^{n+1}$ is a solution of $(\overline{\mathrm{EQ}})$ and $\xi$ is a vector in $\mathbb{R}^{n+1}$, then $\tilde{u}(t, \cdot)=u(t, \cdot)+t \xi$ is also a solution of equation (EQ) with initial data $\tilde{u}(0, \cdot)=u(0, \cdot), \partial_{t} \tilde{u}(0, \cdot)=\partial_{t} u(0, \cdot)+\xi$. This is easy to see since $\frac{d \mu_{t}}{d \widehat{\mu}}(-H+$ $\left.\varrho \operatorname{Vol}(u)^{-1}\right) v$ is translation invariant and $\partial_{t}^{2} \tilde{u}=\partial_{t}^{2} u$. Together with Proposition 2.5 we obtain translating vibrating solutions.

Proposition 2.6. Let $g_{0}=\gamma_{0}^{2} g_{\mathbb{S}^{n}}$ be a spherical metric with $\gamma_{0}>0$ and $d \hat{\mu}$ its surface measure. Let $r_{0}>0, r_{1} \in \mathbb{R}, p, \xi \in \mathbb{R}^{n+1}$. There exists a unique solution $u: \mathbb{R} \times \mathbb{S}^{n} \rightarrow \mathbb{R}^{n+1}$ of (EQ) having the form $u(t, x)=p+r(t) x+t \xi$ with $u(0, x)=p+r_{0} x$ and $\partial_{t} u(0, x)=r_{1} x+\xi$. This solution is the oscillating solution from Proposition 2.5 with initial conditions $r_{0}, r_{1}$ translating with velocity $\xi$. At $t=0$ it is centered at $p$.

\section{Weakly Hyperbolic Linear Systems (WHLS)}

In this section we define weakly hyperbolic linear systems. These systems will arise in the linearization of our equation (EQ) (see page 808). They decompose with respect to time-dependent subbundles into a system of coupled linear wave equations and linear ODEs. We will also allow integrals of the unknowns to appear. After the definition we derive estimates for WHLS, which in a first step are similar to energy estimates for the wave equation that estimate spatial $L^{2}$-Sobolev norms. We then prove the solvability of WHLS. Finally, we integrate our estimates to prove tame estimates for solutions of these systems in the $L^{2}$-Sobolev grading in space and time.

\subsection{Definition of WHLS}

Let $\pi: \mathcal{V} \rightarrow \mathcal{N}$ be a $d$-dimensional Riemannian vector bundle over $\mathcal{N}$. Let F be the Fréchet space $C^{\infty}([0, T] \times \mathcal{N}, \mathcal{V})$ of smooth time-dependent sections of $\mathcal{V}$. Assume that we have an atlas of coordinate charts $\left(x_{\alpha}, U_{\alpha}\right)$ of $\mathcal{N}$ such that $\alpha=1, \ldots, J, x_{\alpha}\left(U_{\alpha}\right)=B_{3}(0)$, and the sets $x_{\alpha}^{-1}\left(B_{1}(0)\right)$ cover $\mathcal{N}$. Assume also that for each such chart there are smooth time-dependent local sections $v_{A}^{(\alpha)}$, $A=1, \ldots, d^{\prime}$, and $\tau_{k}^{(\alpha)}, k=1, \ldots, d^{\prime \prime}$, of $\mathcal{V}\left(d^{\prime}+d^{\prime \prime}=d\right)$ defined on the domain of the chart that together form a basis of the fiber over each point in $U_{\alpha}$. For any other chart $\left(x_{\beta}, U_{\beta}\right)$ with $U_{\alpha} \cap U_{\beta} \neq \varnothing$ we assume that the $v_{A}^{(\alpha)}(p)$ and 
$v_{A}^{(\beta)}(p), p \in U_{\alpha} \cap U_{\beta}$, span the same space and are bases for this space. Furthermore, we assume that the spaces spanned by the $v_{A}^{(\alpha)}$ and the $\tau_{k}^{(\alpha)}$ are orthogonal. If the specific coordinate chart does not play a role or is fixed, then we will omit the index $(\alpha)$. Let $d \hat{\mu}$ be the volume form of a reference metric $g_{0}$. Let $V \in \mathbf{F}$. In each coordinate chart we can decompose

$$
V=V_{\perp}+V_{\mathrm{\top}}:=\phi^{A} v_{A}+\psi^{k} \tau_{k}
$$

We say that $V$ satisfies a weakly hyperbolic linear system if in each coordinate chart $\left(x_{\alpha}, U_{\alpha}\right)$ we have

$$
\begin{aligned}
\partial_{t}^{2} \phi^{A}-L^{A} \phi-N^{A} \psi-Q_{1}^{A} \phi-Q_{2}^{A} \psi & =v^{A}, \\
\partial_{t}^{2} \psi^{k}-M^{k} \psi-P^{k} \phi & =w^{k},
\end{aligned}
$$

for some given $W=v^{A} v_{A}+w^{k} \tau_{k}$. The operators are assumed to be of the following form in local coordinates:

$$
\begin{aligned}
L^{A} \phi & =a^{A i j} \partial_{i} \partial_{j} \phi^{A}+a_{B}^{A i} \partial_{i} \phi^{B}+a_{B}^{A} \phi^{B}+a_{B}^{A 0} \partial_{t} \phi^{B}, \\
N^{A} \psi & =n_{j}^{A i} \partial_{i} \psi^{j}+n_{i}^{A} \psi^{i}+n_{k}^{A 0} \partial_{t} \psi^{k}, \\
Q_{1}^{A} \phi & =q_{1}^{A} \sum_{\beta=1}^{J} \int_{\mathcal{N}} c_{(\beta) B}^{A} \phi_{(\beta)}^{B} d \widehat{\mu}, \\
Q_{2}^{A} \psi & =q_{2}^{A} \sum_{\beta=1}^{J} \int_{\mathcal{N}} b_{(\beta) j}^{A} \psi_{(\beta)}^{j} d \hat{\mu}, \\
M^{k} \psi & =m_{i}^{k} \psi^{i}+m_{i}^{k 0} \partial_{t} \psi^{i}, \\
P^{k} \phi & =p_{B}^{k j} \partial_{j} \phi^{B}+p_{B}^{k 0} \partial_{t} \phi^{B} .
\end{aligned}
$$

Of course we do not apply the summation convention for the index $A$ here. We assume all coefficients and also $v^{A}$ and $w^{k}$ to be smooth functions on $x_{\alpha}\left(U_{\alpha}\right)$ and $\Lambda \delta^{i j} \xi_{i} \xi_{j} \geq a^{A i j} \xi_{i} \xi_{j} \geq \lambda \delta^{i j} \xi_{i} \xi_{j}$ for all $\xi \in \mathbb{R}^{n}$ with some fixed $\Lambda, \lambda>0$. Assume further $a^{A i j}=a^{A j i}, \operatorname{supp} b_{(\beta) j}^{A} \subset x_{\beta}^{-1}\left(B_{2}(0)\right)$, and $\operatorname{supp} c_{(\beta) B}^{A} \subset$ $x_{\beta}^{-1}\left(B_{2}(0)\right)$. Furthermore, we want that the operators are coordinate invariant under coordinate transformations on $\mathcal{N}$ and under a change of basis between different $\left(v_{A}^{(\alpha)}, \tau_{k}^{(\alpha)}\right)$ and $\left(v_{A}^{(\beta)}, \tau_{k}^{(\beta)}\right)$. This implies that $W=v^{A} v_{A}+w^{k} \tau_{k}$ is an element of $\mathcal{V}$. 


\subsection{Norms}

We wish to estimate the components $\phi^{A}, \psi^{k}$ of a solution $V=\phi^{A} v_{A}+\psi^{k} \tau_{k}$ to the WHLS 3.1) with respect to the time-dependent frames $v_{A}, \tau_{k}$. Therefore we introduce the following notation: Let $H^{s}\left(\Omega, \mathbb{R}^{d^{\prime}}\right)$ denote the $L^{2}$-Sobolev space of functions from an open bounded set $\Omega \subset \mathbb{R}^{n}$ to $\mathbb{R}^{d^{\prime}}$. Set $H^{s}(\Omega)=$ $H^{s}(\Omega, \mathbb{R})$. Now the set of functions $\left(\phi_{(\alpha)}^{A}\right)$ can be considered as an element of $H^{s}\left(B_{2}(0), \mathbb{R}^{d^{\prime}}\right)^{\times J}$ with norm

$$
\|\phi\|_{s}=\sum_{\alpha=1}^{J} \sum_{A=1}^{d^{\prime}}\left\|\phi_{(\alpha)}^{A}\right\|_{H^{s}\left(B_{2}(0)\right)} .
$$

We will also have to include $v_{A}, \tau_{k}$ in our estimates and put

$$
\|v\|_{s}=\sum_{\alpha=1}^{J} \sum_{A=1}^{d^{\prime}}\left\|v_{A}^{(\alpha)} \circ x_{\alpha}^{-1}\right\|_{H^{s}\left(B_{2}(0)\right)} .
$$

We similarly define $\|\cdot\|_{C^{s}},\|\| \cdot\|\|_{s}$, and \|\|$\cdot\|\|_{C^{s}}$ as the spatial $C^{s}$-norm, the $L^{2}$ Sobolev norm of order $s$ in space and time, and the $C^{s}$-norm in space and time, respectively.

For a linear differential operator we always define its "norm" to be the norm of the coefficients in local coordinates. For example, if in a local coordinate chart $\left(x_{\alpha}, U_{\alpha}\right)$ we have $L \phi=a^{i j} \partial_{i} \partial_{j} \phi+a^{i} \partial_{i} \phi+a \phi$, then we define the local norm

$$
[L]_{s, \alpha}=\sum_{i, j}\left\|a^{i j}\right\|_{H^{s}\left(B_{2}(0)\right)}+\sum_{i}\left\|a^{i}\right\|_{H^{s}\left(B_{2}(0)\right)}+\|a\|_{H^{s}\left(B_{2}(0)\right)}
$$

and the full norm

$$
[L]_{S}=\sum_{\alpha=1}^{J}[L]_{S, \alpha} .
$$

We define similarly $[L]_{C^{s}},|[L]|_{s}$, and $|[L]|_{C^{s}}$ to measure the coefficients in $\|\cdot\|_{C^{s}}$, $\||\cdot|\|_{s}$, and $\|\mid \cdot\| \|_{C s}$, respectively. Note that these are not the usual operator norms. We also apply this notation for estimates on the integral operators although this does not define a norm, e.g.,

$$
\left[Q_{1}\right]_{S}=\sum_{\alpha=1}^{J} \sum_{A}\left\|q_{1(\alpha)}^{A}\right\|_{H^{s}\left(B_{2}(0)\right)}+\sum_{\alpha=1}^{J} \sum_{A, B}\left\|c_{(\beta) B}^{A}\right\| .
$$

\subsection{Wave and ODE Estimates}

The following $L^{2}$-energy estimate for linear wave equations is standard, but we will need a version that accounts for the finite speed of propagation and keeps the exponential under the integral. The proof uses standard methods and is omitted. For a constant $\Lambda>0$ and $\left(t_{0}, x_{0}\right) \in \mathbb{R}^{n+1}$, we denote $S_{t}\left(t_{0}, x_{0}\right)=\left\{x \in \mathbb{R}^{n}\right.$ : 
$\left.\left|x-x_{0}\right|<\sqrt{\Lambda}\left(t_{0}-t\right)\right\}$. If there is no confusion about the point $\left(t_{0}, x_{0}\right)$, we only write $S_{t}$.

PROPOSITION 3.1. Let $\Omega \subset \mathbb{R}^{n}$ be open and let $\phi:[0, T] \times \Omega \rightarrow \mathbb{R}$ satisfy

$$
\partial_{t}^{2} \phi(t, x)-a^{i j}(t, x) \partial_{i} \partial_{j} \phi(t, x)-a^{i}(t, x) \partial_{i} \phi(t, x)-a(t, x) \phi(t, x)=F(t, x)
$$

where $a^{i j}$ is symmetric and satisfies

$$
\lambda \delta^{i j} \xi_{i} \xi_{j} \leq a^{i j} \xi_{i} \xi_{j} \leq \Lambda \delta^{i j} \xi_{i} \xi_{j}
$$

for constants $\lambda, \Lambda>0$. Furthermore, let $a^{i j}, a^{k}, a$, and $F$ be smooth functions with

$$
1+\sum_{\alpha=0}^{n} \sum_{i, j=1}^{n}\left\|\partial_{\alpha} a^{i j}\right\|_{C^{0}(\Omega)}+\sum_{k=1}^{n}\left\|a^{k}\right\|_{C^{0}(\Omega)}+\|a\|_{C^{0}(\Omega)} \leq K
$$

for some $K>0$. Let $\left(t_{0}, x_{0}\right) \in[0, T] \times \Omega$ such that $S_{0}=S_{0}\left(t_{0}, x_{0}\right) \subset \Omega$. Then there is a constant $C$ depending on $\lambda, \Lambda$, and $K$ such that for $t \in\left[0, t_{0}\right]$

$$
\begin{aligned}
& \|D \phi(t)\|_{L^{2}\left(S_{t}\right)}+\left\|\partial_{t} \phi(t)\right\|_{L^{2}\left(S_{t}\right)}+\|\phi(t)\|_{L^{2}\left(S_{t}\right)} \leq \\
& \qquad e^{C t}\left(\|D \phi(0)\|_{L^{2}\left(S_{0}\right)}+\left\|\partial_{t} \phi(0)\right\|_{L^{2}\left(S_{0}\right)}\right. \\
& \left.\quad+\|\phi(0)\|_{L^{2}\left(S_{0}\right)}+\int_{0}^{t} e^{-C t^{\prime}}\left\|F\left(t^{\prime}\right)\right\|_{L^{2}\left(S_{t^{\prime}}\right)} d t^{\prime}\right) .
\end{aligned}
$$

We need the following ODE estimate:

LEMmA 3.2. If $\Omega \subset \mathbb{R}^{n}$ is open and bounded and $\psi:[0, T] \times \Omega \rightarrow \mathbb{R}^{d}$ is smooth and satisfies $\partial_{t}^{2} \psi^{k}=w^{k}$ for some smooth $w^{k}:[0, T] \times \Omega \rightarrow \mathbb{R}^{d}$, then

$$
\begin{array}{r}
\|\psi(t)\|_{H^{1}(\Omega)}+\left\|\partial_{t} \psi(t)\right\|_{H^{1}(\Omega)} \leq C e^{C t}\left(\|\psi(0)\|_{H^{1}(\Omega)}+\left\|\partial_{t} \psi(0)\right\|_{H^{1}(\Omega)}\right. \\
\left.+\int_{0}^{t} e^{-C t^{\prime}}\left\|w\left(t^{\prime}\right)\right\|_{H^{1}(\Omega)} d t^{\prime}\right)
\end{array}
$$

ProOF. Define for some $\varepsilon>0$

$$
E_{\varepsilon}(t)=\frac{1}{2} \int_{\Omega}|\psi|^{2}+\left|\partial_{t} \psi\right|^{2}+|D \psi|^{2}+\left|\partial_{t} D \psi\right|^{2} d x+\varepsilon
$$

and estimate using Hölder's inequality and Cauchy's inequality

$$
\partial_{t} E_{\varepsilon}(t)^{\frac{1}{2}} \leq C\left(E_{\varepsilon}(t)^{\frac{1}{2}}+\|w(t)\|_{H^{1}(\Omega)}\right) .
$$

Now apply Gronwall's inequality and let $\varepsilon \rightarrow 0$. 


\subsection{Basic Estimates for WHLS}

Define the total energy of the system (3.1) as

$$
\begin{aligned}
E_{S}(t)= & \left\|\partial_{t}^{2} \phi(t)\right\|_{s}+\left\|\partial_{t} \phi(t)\right\|_{s+1}+\|\phi(t)\|_{s+1} \\
& +\left\|\partial_{t} \psi(t)\right\|_{s+1}+\|\psi(t)\|_{s+1} .
\end{aligned}
$$

PROPOSITION 3.3. Assume that $\phi, \psi$ satisfy the weakly hyperbolic linear system (3.1) on a time interval $[0, T]$ and that for some $s \geq\left\lfloor\frac{n}{2}\right\rfloor+2$ and $K_{1}, K_{2}, \lambda_{1}>0$

$$
\begin{aligned}
1+[L]_{s}+\left[\partial_{t} L\right]_{s}+[M]_{s+1}+\left[Q_{1}\right]_{s}+\left[\partial_{t} Q_{1}\right]_{s} \\
+\left[Q_{2}\right]_{s}+\left[\partial_{t} Q_{2}\right]_{s}+[N]_{s}+\left[\partial_{t} N\right]_{s}+[P]_{s+1} \leq K_{2},
\end{aligned}
$$

and

$$
\operatorname{det}\left(\left\langle v_{A}, v_{B}\right\rangle\right)>\lambda_{1}, \quad \operatorname{det}\left(\left\langle\tau_{k}, \tau_{l}\right\rangle\right)>\lambda_{1}
$$

Then we have the estimate

$$
E_{S}(t) \leq C e^{C t} E_{S}(0)+C \int_{0}^{t} e^{C\left(t-t^{\prime}\right)}\left(\left\|v\left(t^{\prime}\right)\right\|_{s}+\left\|\partial_{t} v\left(t^{\prime}\right)\right\|_{s}+\left\|w\left(t^{\prime}\right)\right\|_{s+1}\right) d t^{\prime},
$$

where $C$ only depends on $K_{1}, K_{2}, \lambda, \lambda_{1}, \Lambda$, and $s$.

\section{PROOF.}

(1) Set $B_{1}=B_{1}(0) \subset \mathbb{R}^{n}$ and $B_{2}=B_{2}(0) \subset \mathbb{R}^{n}$. Choose $\left(t_{0}, x_{0}\right)=$ $\left(\frac{3}{2 \sqrt{\Lambda}}, 0\right)$. Recall from Section 3.3 that $S_{t}=\left\{x \in \mathbb{R}^{n}:\left|x-x_{0}\right|<\sqrt{\Lambda}\left(t_{0}-t\right)\right\}$. To avoid constants depending on $S_{t}$ when we apply the elliptic regularity estimate, define $\Omega=B_{7 / 4}(0)$. Then we always have $B_{1} \subset S_{t} \subset \Omega \Subset B_{2}$ for $t \leq t^{*}:=$ $\frac{1}{2 \sqrt{\Lambda}}$. We will first prove the estimate for $t \leq t^{*}$.

(2) Let $\beta$ be a multiindex with $1 \leq|\beta| \leq s$ and $\partial^{\beta}$ be a spatial derivative. Note that by the Sobolev embedding theorem we have $\|\cdot\|_{C^{k}} \leq C\|\cdot\|_{\left\lfloor\frac{n}{2}\right\rfloor+1+k}$. Differentiating the system (3.1) in a coordinate chart yields

$$
\begin{aligned}
\partial_{t}^{2} \partial^{\beta} \phi-L^{A} \partial^{\beta} \phi= & \partial^{\beta} v^{A}+\partial^{\beta}\left(L^{A} \phi\right)-L^{A} \partial^{\beta} \phi+\partial^{\beta}\left(N^{A} \psi\right) \\
& +\partial^{\beta}\left(Q_{1}^{A} \phi\right)+\partial^{\beta}\left(Q_{2}^{A} \phi\right)=: \widetilde{v}^{A} \\
\partial_{t}^{2} \partial^{\beta} \psi^{k}= & \partial^{\beta} w^{k}+\partial^{\beta}\left(M^{k} \psi\right)+\partial^{\beta}\left(P^{k} \phi\right)=: \widetilde{w}^{k} .
\end{aligned}
$$

We want to apply the basic energy estimate Proposition 3.1 and the ODE estimate Lemma 3.2 to this system, and hence we must estimate the terms $\|\widetilde{v}\|_{L^{2}\left(S_{t^{\prime}}\right)}$ and $\|\widetilde{w}\|_{H^{1}(\Omega)}$. We can do this using the Moser inequalities [11, chap. 13, prop. 3.7], the Sobolev embedding theorem, and the assumptions, e.g.,

$$
\begin{aligned}
\left\|\partial^{\beta}(N \psi)\right\|_{L^{2}\left(B_{2}\right)} \leq & C\left([N]_{S}\left(\|\psi\|_{C^{1}}+\left\|\partial_{t} \psi\right\|_{C^{0}}\right)\right. \\
& \left.+[N]_{C^{0}}\left(\|\psi\|_{s+1}+\left\|\partial_{t} \psi\right\|_{s}\right)\right) \\
\leq C & \left(\|\psi\|_{s+1}+\left\|\partial_{t} \psi\right\|_{s+1}\right),
\end{aligned}
$$




$$
\begin{aligned}
\left\|\partial^{\beta}(L \phi)-L \partial^{\beta} \phi\right\|_{L^{2}\left(B_{2}\right)} \leq & C\left([L]_{H^{s}\left(B_{2}\right)}\|\phi\|_{C^{2}\left(B_{2}\right)}\right. \\
& \left.+[L]_{C^{1}\left(B_{2}\right)}\|\phi\|_{H^{s+1}\left(B_{2}\right)}\right) \\
\leq C & \|\phi\|_{H^{s+1}\left(B_{2}\right)} .
\end{aligned}
$$

The other terms are estimated similarly, and we obtain

$$
\begin{aligned}
& \|\tilde{v}\|_{L^{2}\left(S_{t^{\prime}}\right)} \leq C\left(\|v\|_{S}+E_{S}\right), \\
& \|\tilde{w}\|_{H^{1}(\Omega)} \leq C\left(\|w\|_{s+1}+\|\phi\|_{H^{s+2}(\Omega)}+E_{S}\right) .
\end{aligned}
$$

In order to estimate $\|\phi\|_{H^{s+2}(\Omega)}$ we take some multiindex $\beta^{\prime}$ with $\left|\beta^{\prime}\right|=s$. By the elliptic regularity estimate [3, theorem 1, 6.3.1] and the equation (3.1) for $L \phi$, we can estimate

$$
\begin{aligned}
\left\|\partial^{\beta^{\prime}} \phi\right\|_{H^{2}(\Omega)} & \leq C\left(\left\|L \partial^{\beta^{\prime}} \phi\right\|_{L^{2}\left(B_{2}\right)}+\|\phi\|_{s+1}\right) \\
& \leq C\left(\|L \phi\|_{s}+\left\|\partial^{\beta^{\prime}}(L \phi)-L \partial^{\beta^{\prime}} \phi\right\|_{L^{2}\left(B_{2}\right)}+\|\phi\|_{s+1}\right) \\
& \leq C\left(\|v\|_{s}+E_{s}\right) .
\end{aligned}
$$

Applying the basic energy estimate Proposition 3.1 to 3.5a and the ODE estimate Lemma 3.2 to 3.5b), we obtain

$$
\begin{aligned}
&\left\|\partial_{t} \partial^{\beta} \phi^{A}(t)\right\|_{L^{2}\left(B_{1}\right)}+\left\|D \partial^{\beta} \phi^{A}(t)\right\|_{L^{2}\left(B_{1}\right)}+\left\|\partial^{\beta} \phi^{A}(t)\right\|_{L^{2}\left(B_{1}\right)} \leq \\
& C e^{C t} E_{S}(0)+C \int_{0}^{t} e^{C\left(t-t^{\prime}\right)}\left(\left\|v\left(t^{\prime}\right)\right\|_{s}+E_{S}\left(t^{\prime}\right)\right) d t^{\prime}
\end{aligned}
$$

and

$$
\begin{aligned}
& \left\|\partial^{\beta} \psi^{k}(t)\right\|_{H^{1}\left(B_{1}\right)}+\left\|\partial^{\beta} \partial_{t} \psi^{k}(t)\right\|_{H^{1}\left(B_{1}\right)} \leq \\
& \quad C e^{C t} E_{S}(0)+C \int_{0}^{t} e^{C\left(t-t^{\prime}\right)}\left(\left\|w\left(t^{\prime}\right)\right\|_{s+1}+\left\|v\left(t^{\prime}\right)\right\|_{s}+E_{S}\left(t^{\prime}\right)\right) d t^{\prime} .
\end{aligned}
$$

(3) We also need the terms $\left\|\partial_{t}^{2} \phi\right\|_{H^{s}\left(B_{1}\right)}+\left\|\partial_{t} \phi\right\|_{H^{s+1}\left(B_{1}\right)}$ on the left-hand side of the estimate in order to use Gronwall's inequality later since they appear in the energy. Therefore we differentiate the wave part 3.5a additionally with respect to time

$$
\begin{aligned}
\partial_{t}^{2} \partial_{t} \partial^{\beta} & \phi^{A}-L^{A} \partial_{t} \partial^{\beta} \phi \\
= & \partial_{t} \partial^{\beta} v^{A}+\partial^{\beta}\left(\partial_{t} L^{A} \phi\right)+\partial^{\beta}\left(L^{A} \partial_{t} \phi\right)-L^{A} \partial_{t} \partial^{\beta} \phi \\
& +\partial^{\beta}\left(\partial_{t} Q_{1}^{A} \phi\right)+\partial^{\beta}\left(Q_{1}^{A} \partial_{t} \phi\right)+\partial^{\beta}\left(\partial_{t} Q_{2}^{A} \psi\right) \\
& +\partial^{\beta}\left(Q_{2}^{A} \partial_{t} \psi\right)+\partial^{\beta}\left(\partial_{t} N^{A} \psi\right)+\partial^{\beta}\left(N^{A} \partial_{t} \psi\right)=: \widetilde{\widetilde{v}}^{A} .
\end{aligned}
$$

We estimate similarly as above

$$
\| \tilde{\widetilde{v}}_{L^{2}\left(B_{2}\right)} \leq C\left(\left\|\partial_{t} v\right\|_{s}+\|\phi\|_{H^{s+2}(\Omega)}+\left\|\partial_{t}^{2} \psi\right\|_{H^{s}\left(B_{2}\right)}+E_{S}\right) .
$$

For $\|\phi\|_{H^{s+2}(\Omega)}$ we use 3.8 . We use the equation for $\partial_{t}^{2} \psi$ to estimate

$$
\left\|\partial_{t}^{2} \psi\right\|_{H^{s}\left(B_{2}\right)} \leq C\left(\|w\|_{s}+E_{s}\right)
$$


Applying Proposition 3.1 to 3.11, we obtain

$$
\begin{aligned}
& \text { (3.12) }\left\|\partial_{t}^{2} \partial^{\beta} \phi(t)\right\|_{L^{2}\left(B_{1}\right)}+\left\|D \partial_{t} \partial^{\beta} \phi(t)\right\|_{L^{2}\left(B_{1}\right)}+\left\|\partial_{t} \partial^{\beta} \phi(t)\right\|_{L^{2}\left(B_{1}\right)} \leq \\
& C e^{C t} E_{S}(0)+C \int_{0}^{t} e^{C\left(t-t^{\prime}\right)}\left(\left\|\partial_{t} v\left(t^{\prime}\right)\right\|_{s}+\left\|v\left(t^{\prime}\right)\right\|_{s}+\left\|w\left(t^{\prime}\right)\right\|_{s}+E_{S}\left(t^{\prime}\right)\right) d t^{\prime} .
\end{aligned}
$$

(4) We now sum estimates (3.9), (3.10), and (3.12) over all coordinate charts and all $\beta$. The case $\beta=0$ works the same except that the term estimated in (3.7) vanishes. Due to the assumed bounds (3.3) and (3.4) on $v$ and $\tau$, we can compare norms taken on $B_{1}$ and norms taken on $B_{2}$. We can then apply Gronwall's lemma to obtain our estimate for $t \leq \frac{1}{2 \sqrt{\Lambda}}$. The estimate for arbitrary $t$ follows by an iteration.

PROPOSITION 3.4. Assume that $\phi, \psi$ satisfy the weakly hyperbolic system (3.1) on a time interval $[0, T]$ and that for some $K_{1}, K_{2}, \lambda_{1}>0$

$$
\begin{gathered}
\|v\|_{C^{0}}+\left\|\partial_{t} v\right\|_{C^{0}}+\left\|\partial_{t}^{2} \nu\right\|_{C^{0}}+\|\tau\|_{C^{0}}+\left\|\partial_{t} \tau\right\|_{C^{0}} \leq K_{1} \\
1+[L]_{C^{1}}+\left[\partial_{t} L\right]_{C^{0}}+[M]_{C^{1}}+\left[Q_{1}\right]_{C^{0}}+\left[\partial_{t} Q_{1}\right]_{C^{0}} \\
+\left[Q_{2}\right]_{C^{0}}+\left[\partial_{t} Q_{2}\right]_{C^{0}}+[N]_{C^{0}}+\left[\partial_{t} N\right]_{C^{0}}+[P]_{C^{1}} \leq K_{2}
\end{gathered}
$$

and

$$
\operatorname{det}\left(\left\langle v_{A}, v_{B}\right\rangle\right)>\lambda_{1}, \quad \operatorname{det}\left(\left\langle\tau_{k}, \tau_{l}\right\rangle\right)>\lambda_{1}
$$

Suppose further

$$
\|\phi\|_{C^{2}}+\left\|\partial_{t} \phi\right\|_{C^{2}}+\|\psi\|_{C^{1}}+\left\|\partial_{t} \psi\right\|_{C^{1}} \leq K_{3}
$$

and

$$
\|v\|_{C^{0}}+\|w\|_{C^{0}} \leq K_{4}
$$

for some $K_{3}, K_{4}>0$. Then for any $s \geq 0$ we have the estimate

$$
\begin{aligned}
E_{s}(t) \leq & C e^{C t} \sup _{[0, t]}\left(\left\|\partial_{t}^{2} v\right\|_{s}+\left\|\partial_{t} v\right\|_{s+1}+\|v\|_{s+1}+\|\tau\|_{s+1}+\left\|\partial_{t} \tau\right\|_{s+1}+1\right) \\
& +C e^{C t} E_{s}(0) \\
& +C \int_{0}^{t} e^{C\left(t-t^{\prime}\right)}\left(\|v\|_{s}+\left\|\partial_{t} v\right\|_{s}+\|w\|_{s+1}+[L]_{s}\right. \\
& +\left[\partial_{t} L\right]_{s}+\left[Q_{1}\right]_{s}+\left[\partial_{t} Q_{1}\right]_{s}+\left[Q_{2}\right]_{s}+\left[\partial_{t} Q_{2}\right]_{s} \\
& \left.+[N]_{s}+\left[\partial_{t} N\right]_{s}+[M]_{s+1}+[P]_{s+1}\right) d t^{\prime}
\end{aligned}
$$

where $C$ depends only on $K_{1}, K_{2}, K_{3}, K_{4}, \lambda, \lambda_{1}, \Lambda$, and $s$. 
PROOF. The strategy of the proof is very similar to the proof of Proposition 3.3. We just use the assumptions (3.13) and (3.14) instead of the Sobolev embedding theorem in our estimates, e.g., replace (3.6) and 3.7p by

$$
\begin{aligned}
\left\|\partial^{\beta}(L \phi)-L \partial^{\beta} \phi\right\|_{L^{2}\left(B_{2}\right) \leq} & C\left([L]_{H^{s}\left(B_{2}\right)}\|\phi\|_{C^{2}\left(B_{2}\right)}\right. \\
& \left.+[L]_{C^{1}\left(B_{2}\right)}\|\phi\|_{H^{s+1}\left(B_{2}\right)}\right) \\
\leq & C\left([L]_{H^{s}\left(B_{2}\right)}+\|\phi\|_{H^{s+1}\left(B_{2}\right)}\right), \\
\left\|\partial^{\beta}(N \psi)\right\|_{L^{2}\left(B_{2}\right) \leq} & C\left([N]_{s}\left(\|\psi\|_{C^{1}}+\left\|\partial_{t} \psi\right\|_{C^{0}}\right)\right. \\
& \left.+[N]_{C^{0}}\left(\|\psi\|_{s+1}+\left\|\partial_{t} \psi\right\|_{s}\right)\right) \\
\leq & C\left([N]_{s}+\|\psi\|_{s+1}+\left\|\partial_{t} \psi\right\|_{s}\right) .
\end{aligned}
$$

The term

$$
\sup _{[0, t]}\left(\left\|\partial_{t}^{2} \nu\right\|_{s}+\left\|\partial_{t} v\right\|_{s+1}+\|v\|_{s+1}+\|\tau\|_{s+1}+\left\|\partial_{t} \tau\right\|_{s+1}+1\right)
$$

arises when we compare norms taken on $B_{1}$ with norms taken on $B_{2}$ since we do not assume that we have bounds on these terms here.

Remark 3.5. As said in the last lines of the previous proof, if we had a bound

$$
\|v\|_{s}+\left\|\partial_{t} v\right\|_{s}+\left\|\partial_{t}^{2} v\right\|_{s}+\|\tau\|_{s}+\left\|\partial_{t} \tau\right\|_{s} \leq C_{s}
$$

for all $s>0$ and constants $C_{s}>0$, we could remove the term 3.16 from the estimate.

If we do not use the assumption $\|\phi\|_{C^{2}}+\left\|\partial_{t} \phi\right\|_{C^{2}} \leq K_{3}$ in (3.15) and in the related estimates and assume $P=0, Q_{1}=0, Q_{2}=0$, and $M=0$, we obtain the estimate

$$
\begin{array}{r}
E_{S}(t) \leq C e^{C t} E_{S}(0)+C \int_{0}^{t} e^{C\left(t-t^{\prime}\right)}\left(\|v\|_{s}+\left\|\partial_{t} v\right\|_{S}+\|w\|_{s+1}+[L]_{S}\|\phi\|_{C^{2}}\right. \\
\left.+\left[\partial_{t} L\right]_{S}\|\phi\|_{C^{2}}+[L]_{s}\left\|\partial_{t} \phi\right\|_{C^{2}}\right) d t^{\prime}
\end{array}
$$

We will apply this modified estimate to estimate the time of existence in Section 6 . The idea behind this is that $[L]_{s}+\left[\partial_{t} L\right]_{s}$ might not be small and so it needs a factor that is small if $\phi$ is small.

\subsection{Solvability of WHLS}

Proposition 3.6. Let $V_{0}, V_{1} \in C^{\infty}(\mathcal{N}, \mathcal{V})$ and $W \in C^{\infty}([0, T] \times \mathcal{N}, \mathcal{V})$ be given. Then the system (3.1) has a unique smooth solution $V$ on $[0, T] \times \mathcal{N}$ with $V(0)=V_{0}$ and $\partial_{t} V(0)=V_{1}$.

Proof. Write locally $V=\phi^{A} v_{A}+\psi^{k} \tau_{k}$ and $W=v^{A} v_{A}+w^{k} \tau_{k}$. Since

$$
\partial_{t} V(0)=\partial_{t} \phi^{A}(0) v_{A}(0)+\partial_{t} \psi^{k}(0) \tau_{k}(0)+\phi^{A}(0) \partial_{t} v_{A}(0)+\psi^{k}(0) \partial_{t} \tau_{k}(0)
$$


our initial conditions in terms of $\phi_{0}^{A}:=\phi^{A}(0), \psi_{0}^{k}:=\psi^{k}(0), \phi_{1}^{A}:=\partial_{t} \phi^{A}(0)$, and $\psi_{1}^{k}:=\partial_{t} \psi^{k}(0)$ are $\phi_{0}^{A}=\left\langle V_{0}, v_{B}(0)\right\rangle v^{B A}(0), \psi_{0}^{k}=\left\langle V_{0}, \tau_{l}(0)\right\rangle \tau^{k l}(0)$, and

$$
\begin{aligned}
\phi_{1}^{A}= & \left\langle V_{1}, v_{B}(0)\right\rangle v^{B A}(0)-\phi_{0}^{C}\left\langle\partial_{t} \nu_{C}(0), v_{B}(0)\right\rangle v^{B A}(0) \\
& -\psi_{0}^{k}\left\langle\partial_{t} \tau_{k}(0), v_{B}(0)\right\rangle v^{B A}(0), \\
\psi_{1}^{k}= & \left\langle V_{1}, \tau_{l}(0)\right\rangle \tau^{l k}(0)-\phi_{0}^{A}\left\langle\partial_{t} v_{A}(0), \tau_{l}(0)\right\rangle \tau^{l k}(0) \\
- & \psi_{0}^{m}\left\langle\partial_{t} \tau_{m}(0), \tau_{l}(0)\right\rangle \tau^{l k}(0),
\end{aligned}
$$

with $v^{A B}=\left(\left\langle v_{A}, v_{B}\right\rangle\right)^{-1}$ and $\tau^{k l}=\left(\left\langle\tau_{k}, \tau_{l}\right\rangle\right)^{-1}$.

We will solve the system (3.1) for $\phi^{A}, \psi^{k}$ by a simple fixed point iteration. Start with $\phi_{(0)}^{A}=0, \psi_{(0)}^{k}=0$. Then solve inductively

$$
\begin{aligned}
\partial_{t}^{2} \phi_{(m+1)}^{A}-L^{A} \phi_{(m+1)} & =v^{A}+N^{A} \psi_{(m)}+Q_{1}^{A} \phi_{(m)}+Q_{2}^{A} \psi_{(m)}, \\
\partial_{t}^{2} \psi_{(m+1)}^{k} & =w^{k}+M^{k} \psi_{(m)}+P^{k} \phi_{(m)},
\end{aligned}
$$

with initial conditions

$$
\begin{aligned}
\phi_{(m+1)}^{A}(0) & =\phi_{0}^{A}, & \psi_{(m+1)}^{k}(0) & =\psi_{0}^{k}, \\
\partial_{t} \phi_{(m+1)}^{A}(0) & =\phi_{1}^{A}, & \partial_{t} \psi_{(m+1)}^{k}(0) & =\psi_{1}^{k} .
\end{aligned}
$$

The system (3.18) only consists of linear wave equations for $\phi_{(m+1)}^{A}$ and linear ODEs for $\psi_{(m+1)}^{k}$. The ODEs have a unique smooth solution on $[0, T]$. The wave equations can be solved locally in space and for a short time due to finite speed of propagation. The coordinate invariance of the system implies that $V_{(m+1)}=$ $\phi_{(m+1)}^{A} \nu_{A}+\psi_{(m+1)}^{k} \tau_{k}$ is well defined for small $t$. This can be iterated such that we get a solution on $[0, T]$.

The differences $\widetilde{\phi}_{(m+1)}^{A}=\phi_{(m+1)}^{A}-\phi_{(m)}^{A}$ and $\widetilde{\psi}_{(m+1)}^{k}=\psi_{(m+1)}^{k}-\psi_{(m)}^{k}$ satisfy a WHLS. Using Proposition 3.3 it is easy to check that the iteration converges to a unique smooth solution.

\subsection{Tame Estimate for WHLS}

Proposition 3.7. Let the assumptions of Proposition 3.3 be satisfied with $s_{0} \geq$ $\left\lfloor\frac{n}{2}\right\rfloor+2$. Let locally $V=\phi^{A} v_{A}+\psi^{k} \tau_{k}$ and $W=v^{A} v_{A}+w^{k} \tau_{k}$. Let $V(0)=V_{0}$, $\partial_{t} V(0)=V_{1}$ with

$$
\left\|V_{0}\right\|_{s_{0}+2}+\left\|V_{1}\right\|_{s_{0}+1} \leq K_{3}
$$

for some $K_{3}>0$. Suppose further

$$
\|v\|_{s_{0}+2} \leq K_{2}^{\prime} \quad \text { and } \quad\|W\|_{s_{0}+1}+\left\|\partial_{t} W\right\|_{s_{0}} \leq K_{4}
$$


for some $K_{2}^{\prime}, K_{4}>0$. Then for any $s \geq 1$ we have the estimate

$$
\begin{aligned}
& \||V|\|_{s} \leq C\left(\left\|V_{0}\right\|_{s+1}+\left\|V_{1}\right\|_{s}+\|\| W\left\|_{\left\lfloor\frac{n}{2}\right\rfloor+s}+\right\|\left[\left.L\right|_{\left\lfloor\frac{n}{2}\right\rfloor+s}+|[M]|_{s}\right.\right. \\
& +|[N]|_{\left\lfloor\frac{n}{2}\right\rfloor+s}+|[P]|_{s}+\mid\left[Q_{1}\right]_{\left\lfloor\frac{n}{2}\right\rfloor+s} \\
& +\left|\left[Q_{2}\right]_{\left\lfloor\frac{n}{2}\right\rfloor+s}+\left\||| v\left|\left\|_{s+\left\lfloor\frac{n}{2}\right\rfloor+2}+\right\|\right||\tau|\right\|_{s+\left\lfloor\frac{n}{2}\right\rfloor+2}+1\right)
\end{aligned}
$$

with $C$ depending on $K_{1}, K_{2}, K_{3}, K_{4}, \lambda, \lambda_{1}, \Lambda, s$, and $T$.

PROOF.

(1) We first show that the assumptions of Proposition 3.4 are also satisfied. Define $\phi_{0}^{A}=\left\langle V_{0}, v_{B}(0)\right\rangle \nu^{B A}(0), \psi_{0}^{k}=\left\langle V_{0}, \tau_{l}(0)\right\rangle \tau^{l k}(0)$, and

$$
\begin{aligned}
\phi_{1}^{A}= & \left\langle V_{1}, v_{B}(0)\right\rangle v^{B A}(0)-\phi_{0}^{C}\left\langle\partial_{t} v_{C}(0), v_{B}(0)\right\rangle v^{B A}(0) \\
& -\psi_{0}^{k}\left\langle\partial_{t} \tau_{k}(0), v_{B}(0)\right\rangle v^{B A}(0), \\
\psi_{1}^{k}= & \left\langle V_{1}, \tau_{l}(0)\right\rangle \tau^{l k}(0)-\phi_{0}^{A}\left\langle\partial_{t} v_{A}(0), \tau_{l}(0)\right\rangle \tau^{l k}(0) \\
& -\psi_{0}^{j}\left\langle\partial_{t} \tau_{j}(0), \tau_{l}(0)\right\rangle \tau^{l k}(0)
\end{aligned}
$$

(cf. (3.17)). We can represent $v^{A}=v^{A B}\left\langle W, v_{B}\right\rangle$ and $w^{k}=\tau^{k l}\left\langle W, \tau_{l}\right\rangle$. Using first Moser inequalities, the Sobolev embedding theorem, and the assumptions, we obtain the bounds

$$
\begin{array}{r}
\left\|\phi_{0}\right\|_{s_{0}+2}+\left\|\phi_{1}\right\|_{s_{0}+1}+\left\|\psi_{0}\right\|_{s_{0}+1}+\left\|\psi_{1}\right\|_{s_{0}+1} \leq \\
C\left(\left\|V_{0}\right\|_{s_{0}+2}+\left\|V_{1}\right\|_{s_{0}+1}\right) \leq C
\end{array}
$$

and

$$
\|v\|_{s_{0}}+\left\|\partial_{t} v\right\|_{s_{0}}+\|w\|_{s_{0}+1} \leq C\left(\|W\|_{s_{0}+1}+\left\|\partial_{t} W\right\|_{s_{0}}\right) \leq C .
$$

So by the Sobolev embedding theorem and Proposition 3.3 we have the estimate

$$
\|\phi\|_{C^{2}}+\left\|\partial_{t} \phi\right\|_{C^{2}}+\left\|\partial_{t}^{2} \phi\right\|_{C^{1}}+\left\|\partial_{t} \psi\right\|_{C^{1}}+\|\psi\|_{C^{1}} \leq C .
$$

Hence together with the other assumptions and the Sobolev embedding theorem, the assumptions of Proposition 3.4 are satisfied.

(2) We will first show by an induction on $s$ that

$$
\|\phi \mid\|_{s}+\|\psi \psi\|_{s} \leq C R_{S}
$$

with

$$
\begin{aligned}
R_{S}:= & \left\|\phi_{0}\right\|_{s+1}+\left\|\psi_{0}\right\|_{s}+\left\|\phi_{1}\right\|_{s}+\left\|\psi_{1}\right\|_{s}+\|\mid\| v \|_{\left\lfloor\frac{n}{2}\right\rfloor+s} \\
& +\|w\|\left\|_{s}+\right\|\left|\|v\|_{\left\lfloor\frac{n}{2}\right\rfloor+s+2}+\left\|\left|\|\|_{\left\lfloor\frac{n}{2}\right\rfloor+s+2}+\left\|\left.[L]\right|_{\left\lfloor\frac{n}{2}\right\rfloor+s}+\right\|[M]\right|_{s}\right.\right. \\
& \left.+\| N]\left.\right|_{\left\lfloor\frac{n}{2}\right\rfloor+s}+\| P\right]\left.\right|_{s}+\|\left[\left.Q_{1}\right|_{\left\lfloor\frac{n}{2}\right\rfloor+s}+\|\left[\left.Q_{2}\right|_{\left\lfloor\frac{n}{2}\right\rfloor+s}+1 .\right.\right.
\end{aligned}
$$

The case $s=1$ is trivial in view of (3.19), so assume (3.20) for some $s>1$. 
(3) We have to prove the estimate

$$
\int_{0}^{T}\left\|\partial_{t}^{j} \phi\right\|_{s+1-j}^{2} d t+\int_{0}^{T}\left\|\partial_{t}^{j} \psi\right\|_{s+1-j}^{2} d t \leq C R_{s+1}^{2}
$$

for $j=0, \ldots, s+1$. To this end we do an induction on $j$ as long as $j \leq s+1$. For the base cases $j=0$ and $j=1$ we apply Proposition 3.4 .

$$
\begin{gathered}
\left\|\partial_{t}^{2} \phi(t)\right\|_{s}+\left\|\partial_{t} \phi(t)\right\|_{s+1}+\|\phi(t)\|_{s+1}+\left\|\partial_{t} \psi(t)\right\|_{s+1}+\|\psi(t)\|_{s+1} \\
\leq C \sup _{[0, T]}\left(\left\|\partial_{t}^{2} v\right\|_{s}+\left\|\partial_{t} v\right\|_{s+1}+\|v\|_{s+1}+\|\tau\|_{s+1}+\left\|\partial_{t} \tau\right\|_{s+1}+1\right) \\
+C E_{s}(0)+C \int_{0}^{t}\|v\|_{s}+\left\|\partial_{t} v\right\|_{s}+\|w\|_{s+1}+[L]_{s}+\left[\partial_{t} L\right]_{s}+\left[Q_{1}\right]_{s} \\
+\left[\partial_{t} Q_{1}\right]_{s}+\left[Q_{2}\right]_{s}+\left[\partial_{t} Q_{2}\right]_{s}+[N]_{s}+\left[\partial_{t} N\right]_{s} \\
+[M]_{s+1}+[P]_{s+1} d t^{\prime} .
\end{gathered}
$$

We neglect some unnecessary terms on the left-hand side, square, and integrate this estimate.

To estimate $E_{S}(0)$ note that we can estimate $\left\|\partial_{t}^{2} \phi(0)\right\|_{s}$ using the equation by

$$
\begin{aligned}
\left\|\partial_{t}^{2} \phi(0)\right\|_{s} \leq & \|L(0) \phi(0)\|_{s}+\|N(0) \psi(0)\|_{s} \\
& +\left\|Q_{1}(0) \phi(0)\right\|_{s}+\left\|Q_{2}(0) \psi(0)\right\|_{s}+\|v(0)\|_{s} .
\end{aligned}
$$

We then estimate

$$
\begin{aligned}
\|L(0) \phi(0)\|_{s} & \leq C\left([L(0)]_{s}\left\|\phi_{0}\right\|_{C^{2}}+[L(0)]_{C^{0}}\left\|\phi_{0}\right\|_{s+2}\right) \\
& \left.\leq C(\| L] C_{C^{s}}+\left\|\phi_{0}\right\|_{s+2}\right) \\
& \left.\leq\left. C(\| L]\right|_{\left\lfloor\frac{n}{2}\right\rfloor+s+1}+\left\|\phi_{0}\right\|_{s+2}\right) .
\end{aligned}
$$

The other terms can be estimated similarly and hence $E_{S}(0) \leq C R_{s+1}$.

We estimate

$$
\begin{gathered}
\sup _{[0, T]}\left(\left\|\partial_{t}^{2} v\right\|_{s}+\left\|\partial_{t} v\right\|_{s+1}+\|v\|_{s+1}+\|\tau\|_{s+1}+\left\|\partial_{t} \tau\right\|_{s+1}\right) \leq \\
C\left(\|\| v\|\|_{C^{s+2}}+\|\tau\| \|_{C^{s+2}}\right) \leq C\left(\|\| v\left|\left\|_{\left\lfloor\frac{n}{2}\right\rfloor+s+3}+\right\|\|\tau \mid\|_{\left\lfloor\frac{n}{2}\right\rfloor+s+3}\right) .\right.
\end{gathered}
$$

This yields the base cases for the induction on $j$.

(4) Assume that (3.21) holds true for some $1<j<s+1$ and for $j-1$. Using the equations we can write

$$
\begin{aligned}
& \int_{0}^{T}\left\|\partial_{t}^{j+1} \phi\right\|_{s-j}^{2} d t=\int_{0}^{T}\left\|\partial_{t}^{j-1}\left(L \phi+Q_{1} \phi+Q_{2} \psi+N \psi+v\right)\right\|_{s-j}^{2} d t \\
& \int_{0}^{T}\left\|\partial_{t}^{j+1} \psi\right\|_{s-j}^{2} d t=\int_{0}^{T}\left\|\partial_{t}^{j-1}(M \psi+P \phi+w)\right\|_{s-j}^{2} d t .
\end{aligned}
$$


Now it is almost straightforward to estimate each term on the right by $C R_{s+1}^{2}$ using the first Moser inequality in space and time, the assumptions, and the induction hypothesis. The only term that needs more care is $\int_{0}^{T}\left\|\partial_{t}^{j-1}(L \phi)\right\|_{s-j}^{2} d t$ since an application of the Moser inequality in space and time would mix space and time derivatives and leave us with a term $\||\phi|\|_{s+1}^{2}$. But if we first expand $\partial_{t}^{j-1} \partial^{\beta}(L \phi)$ with $|\beta|=s-j$ using the product rule, we see that at most $s-j+2$ space derivatives fall on $\phi$ and $s-j+2<s+1$. We can take out this critical term, which is easy to estimate by

$$
\left.\int_{0}^{T}\left\|L \partial_{t}^{j-1} \partial^{\beta} \phi\right\|_{s-j}^{2} d t \leq C \| L\right]\left\|_{C^{0}}^{2} \int_{0}^{T}\right\| \partial_{t}^{j-1} \phi \|_{s-j+2}^{2} d t \leq C R_{s+1}^{2}
$$

in view of the induction hypothesis (3.21) for $j-1$. The remaining terms can be estimated by $\||\phi|\|_{s}+|[L]|_{S}$ using the Moser inequality if we apply it for $D L$ or $\partial_{t} L$ and $\phi$. This proves 3.20 for any $s>0$.

(5) It is simple to rewrite (3.20) in terms of $V_{0}, V_{1}, V$, and $W$ using the Moser inequalities and the representations $v^{A}=v^{A B}\left\langle W, v_{B}\right\rangle$ and $w^{k}=\tau^{k l}\left\langle W, \tau_{l}\right\rangle$.

\section{Short-Time Existence}

THEOREM 4.1. For every smooth immersion $u_{0}: \mathcal{N} \rightarrow \mathcal{M}$ with $\operatorname{Vol}\left(u_{0}\right)=\operatorname{Vol}_{0}$ $>0$ and initial velocity $u_{1} \in \Gamma\left(u_{0}^{*} T \mathcal{N}\right)$, there exists $\varepsilon>0$ and a smooth family of immersions $u:[0, \varepsilon) \times \mathcal{N} \rightarrow \mathcal{M}$ solving the Cauchy problem

$$
\left\{\begin{array}{l}
\bar{\nabla}_{\partial_{t}} \partial_{t} u=\frac{d \mu_{t}}{d \hat{\mu}}\left(-H(u)+\frac{\varrho}{\operatorname{Vol}(u)}\right) v \text { for all } t \in[0, \varepsilon), \\
u(0)=u_{0}, \\
\partial_{t} u(0)=u_{1} .
\end{array}\right.
$$

Remark 4.2. Here we only prove existence. Uniqueness is a special case of our stability estimate Theorem 6.1 (see Corollary 6.2).

Our equation $(\mathrm{EQ})$ is a quasi-linear second-order partial differential equation. As we will see in the proof, the linearization (4.1) is not strictly hyperbolic. Due to the diffeomorphism invariance of the mean curvature, only the normal part of the linearized operator is a wave operator. For Ricci flow and mean curvature flow, a suitable family of reparametrizations has been used to remove such a degeneracy. This procedure is known as DeTurck's trick [2]. Here this does not work since, due to the $d \hat{\mu}$-term the action is not diffeomorphism invariant and the evolution of the reparametrizations does not decouple from our equation. It is not clear how this degeneracy can be removed. We therefore work directly with the degenerate equation and use the Nash-Moser inverse function theorem to obtain short-time existence. The strategy of the proof is similar to the short-time existence proof for the Ricci flow given by Hamilton in [5]. For background on the Nash-Moser theorem we recommend [4]. 


\subsection{Euclidean Case}

We will first prove Theorem 4.1 for the simpler case $\mathcal{M}=\mathbb{R}^{n+1}$. The modifications necessary to generalize this result to arbitrary target manifolds are indicated in Section 4.2

\section{Proof of Theorem 4.1 (EuClidean CASe).}

(1) The Strategy. Let $\mathbf{F}$ be the Fréchet space $C^{\infty}\left([0, T] \times \mathcal{N}, \mathbb{R}^{n+1}\right)$ and let $\mathbf{F}_{0}$ be the Fréchet space $C^{\infty}\left(\mathcal{N}, \mathbb{R}^{n+1}\right)$. We define the open subsets

$$
\begin{aligned}
\mathbf{U} & =\left\{u \in \mathbf{F}, \operatorname{det}\left(g_{i j}\right)>0 \text { for all } t \in[0, T]\right\}, \\
\mathbf{U}_{0} & =\left\{u \in \mathbf{F}_{0}, \operatorname{det}\left(g_{i j}\right)>0\right\},
\end{aligned}
$$

and on a subset $\mathbf{U}^{\prime} \subset \mathbf{U}$ to be chosen later, we define the operator $\mathfrak{P}: \mathbf{U}^{\prime} \rightarrow \mathbf{F}$ by

$$
\mathfrak{P}(u)=\bar{\nabla}_{\partial_{t}} \partial_{t} u-\frac{d \mu_{t}}{d \widehat{\mu}}\left(-H(u)+\frac{\varrho}{\operatorname{Vol}(u)}\right) v .
$$

Of course, $\mathfrak{P}(u)$ is a vector field along $u$ but we identify $T_{u(x)} \mathbb{R}^{n+1}$ with $\mathbb{R}^{n+1}$ in the usual way.

From the initial data and the equation we can compute all time derivatives that a solution must have at $t=0$. Now by Borel's lemma we can find $\bar{u}:[0, T] \times \mathcal{N} \rightarrow$ $\mathbb{R}^{n+1}$ with these time derivatives and $\bar{u}(0)=u_{0}$. By making $T$ small we can assume that $\mathfrak{P}(\bar{u})$ is defined. Then $\bar{f}:=\mathfrak{P}(\bar{u})$ satisfies $\left.\partial_{t}^{k} \bar{f}\right|_{t=0}=0$ for all $k=0,1, \ldots$

We shall use the Nash-Moser inverse function theorem to show that the operator $\mathscr{P}: \mathbf{U} \rightarrow \mathbf{F} \times \mathbf{U}_{0} \times \mathbf{F}_{0}$ defined by

$$
\mathscr{P}(u)=\left(\mathfrak{P}(u), u(0), \partial_{t} u(0)\right)
$$

is locally invertible in a neighborhood of $\bar{u}$. This implies that there exists a neighborhood $\mathcal{W}$ of $\left(\bar{u}, u_{0}, u_{1}\right)$ such that we can solve $\mathscr{P}(u)=\left(f, \tilde{u}_{0}, \tilde{u}_{1}\right)$ for every $\left(f, \tilde{u}_{0}, \tilde{u}_{1}\right) \in \mathcal{W}$. We put $\bar{f}=0$ for $t<0$ and define $f_{\varepsilon}(t)=\bar{f}(t-\varepsilon)$ for $0 \leq \varepsilon \leq \varepsilon_{0}$. If $\varepsilon$ is small enough such that $\left(f_{\varepsilon}, u_{0}, u_{1}\right) \in \mathcal{W}$, then we get a solution of $\mathfrak{P}(u)=f_{\varepsilon}$ with the right initial conditions. Then in fact $\mathfrak{P}(u)=0$ for $0 \leq t \leq \varepsilon$.

(2) LinEARIZATION. To apply the Nash-Moser inverse function theorem to the operator $\mathscr{P}$, we have to analyze its linearization. Therefore let $V \in \mathbf{F}$ and let $u:[0, T] \times \mathcal{N} \rightarrow \mathbb{R}^{n+1}$ be a smooth family of immersions such that $\operatorname{Vol}(u)>0$. Decompose $V=\phi v+\psi^{k} \partial_{k} u$. Then we can write $D \mathfrak{P}(u) V=W^{0} v+W^{k} \partial_{k} u$ 
with

$$
\begin{aligned}
W^{0}= & \partial_{t}^{2} \phi-\frac{d \mu_{t}}{d \hat{\mu}}\left\{\Delta \phi+|h|^{2} \phi-\langle\nabla H, \psi\rangle-\frac{\varrho}{\operatorname{Vol}(u)^{2}} \int_{\mathcal{N}} \phi d \mu_{t}\right. \\
& \left.+\left(-H+\frac{\varrho}{\operatorname{Vol}(u)}\right)(\operatorname{div} \psi+H \phi)\right\} \\
& +\phi\left\langle\partial_{t}^{2} \nu, v\right\rangle+2 \partial_{t} \psi^{k}\left\langle\partial_{t} \partial_{k} u, v\right\rangle+\psi^{k}\left\langle\partial_{t}^{2} \partial_{k} u, v\right\rangle \\
W^{k}= & \partial_{t}^{2} \psi^{k}+\frac{d \mu_{t}}{d \hat{\mu}}\left(-H+\frac{\varrho}{\operatorname{Vol}(u)}\right)\left(\nabla^{k} \phi-h_{i}{ }^{k} \psi^{i}\right) \\
& +2 \partial_{t} \phi\left\langle\partial_{t} \nu, \partial_{j} u\right\rangle g^{j k}+\phi\left\langle\partial_{t}^{2} \nu, \partial_{j} u\right\rangle g^{j k} \\
& +2 \partial_{t} \psi^{l}\left\langle\partial_{t} \partial_{l} u, \partial_{j} u\right\rangle g^{j k}+\psi^{l}\left\langle\partial_{t}^{2} \partial_{l} u, \partial_{j} u\right\rangle g^{j k} .
\end{aligned}
$$

To see this, let $u_{\varepsilon}$ be a variation of $u$ with $\left.\partial_{\varepsilon}\right|_{\varepsilon=0} u_{\varepsilon}=V$. It is well known that

$$
-\left.\partial_{\varepsilon}\right|_{\varepsilon=0} H\left(u_{\varepsilon}\right)=\Delta\langle V, v\rangle+|h|^{2}\langle V, v\rangle-\left\langle\nabla H, V^{\top}\right\rangle
$$

and

$$
\begin{aligned}
-\left.\partial_{\varepsilon}\right|_{\varepsilon=0} v\left(u_{\varepsilon}\right)=-\left\langle\left.\partial_{\varepsilon}\right|_{\varepsilon=0} v\left(u_{\varepsilon}\right), \partial_{l} u\right\rangle g^{l k} \partial_{k} u & =\left\langle\partial_{l} V, v\right\rangle g^{l k} \partial_{k} u \\
=\partial_{l}\langle V, v\rangle g^{l k} \partial_{k} u-\left\langle V, \partial_{l} v\right\rangle g^{k l} \partial_{k} u & =\nabla\langle V, v\rangle-\left\langle V, \partial_{l} u\right\rangle h^{l k} \partial_{k} u .
\end{aligned}
$$

By [1, lemma 2.1] the variation of $\operatorname{Vol}\left(u_{\varepsilon}\right)^{-1}$ is given by

$$
-\frac{1}{\operatorname{Vol}(u)^{2}} \int_{\mathcal{N}}\langle V, v\rangle d \mu_{t}
$$

and the variation of $d \mu_{t}$ is given by

$$
\left.\frac{\partial}{\partial \varepsilon}\right|_{\varepsilon=0} d \mu\left(u_{\varepsilon}\right)=\left(\langle V, v\rangle H+\operatorname{div} V^{\top}\right) d \mu .
$$

The decomposition of $\partial_{t}^{2} V$ into normal and tangential parts is straightforward.

To check that (4.1) is a WHLS, take the bundle $\mathcal{V}=\mathcal{N} \times \mathbb{R}^{n+1}, d^{\prime}=1, d^{\prime \prime}=n$. Take $v_{1}=v$ and $\tau_{k}=\partial_{k} u$. Let $\eta_{\alpha}$ be a partition of unity subordinate to the sets $x_{\alpha}^{-1}\left(B_{2}(0)\right)$. Then (4.1) is a WHLS with $Q_{2}=0$,

$$
Q_{1} \phi=-\frac{d \mu_{t}}{d \hat{\mu}} \frac{\varrho}{\operatorname{Vol}(u)^{2}} \sum_{\alpha=1}^{J} \int_{\mathcal{N}} \eta_{\alpha} \phi \frac{d \mu_{t}}{d \hat{\mu}} d \hat{\mu}
$$

and obvious definitions of the operators $L, N, M$, and $P$.

(3) Conclusion. Given $\left(W, V_{0}, V_{1}\right)$, the existence of a unique smooth solution $V=\phi v+\psi^{k} \tau_{k}$ to (4.1) satisfying $V(0)=V_{0}, \partial_{t} V(0)=V_{1}$, is assured by Proposition 3.6. Hence $D \mathscr{P}(u)$ is invertible. 
From [4, cor. II.2.2.7] we know that a nonlinear partial differential operator is a smooth tame map. But $\mathfrak{P}$ is not a differential operator in this sense because it includes the $\operatorname{Vol}(u)$-term. But we can write $\mathfrak{P}(u)=\hat{\mathfrak{P}}(u, \operatorname{Vol}(u))$ where $\hat{\mathfrak{P}}$ is a differential operator of second order in $u$ and zeroth order in $\operatorname{Vol}(u)$. The tameness and smoothness of the map $u \mapsto \operatorname{Vol}(u)$ can be checked easily by writing

$$
\operatorname{Vol}(u)=\operatorname{Vol}\left(u_{0}\right)+\int_{0}^{t} \partial_{t} \operatorname{Vol}(u) d t=\operatorname{Vol}\left(u_{0}\right)+\int_{0}^{t} \int_{\mathcal{N}}\left\langle\partial_{t} u, v\right\rangle d \mu_{t} d t .
$$

To make sure that $D \mathscr{P}^{-1}$ satisfies a tame estimate, we will apply Proposition 3.7. We can estimate $\mid[E]_{\left\lfloor\frac{n}{2}\right\rfloor+s} \leq C\left(1+\|\| u \|_{\left\lfloor\frac{n}{2}\right\rfloor+s+3}\right)$ for $E \in\{L, M, N$, $P, Q_{1}, Q_{2}$ \} using the Moser inequalities since at most third derivatives of $u$ occur in the operators. Also, by the third Moser inequality [11, chap. 13, prop. 3.9] if $\operatorname{det} g_{i j}>\lambda>0$

$$
\begin{aligned}
&\|\| v \|_{s+\left\lfloor\frac{n}{2}\right\rfloor+2} \leq C\left(1+\left|\|u \mid\|_{s+\left\lfloor\frac{n}{2}\right\rfloor+3}\right),\right. \\
&\|\| \tau \mid \|_{s+\left\lfloor\frac{n}{2}\right\rfloor+2} \leq C\left(1+\left|\|u \mid\|_{s+\left\lfloor\frac{n}{2}\right\rfloor+3}\right) .\right.
\end{aligned}
$$

Hence Proposition 3.7 provides us with a tame estimate for $D \mathscr{P}^{-1}$ if we just choose a neighborhood around the data such that its assumptions are satisfied. This is always possible due to compactness of the domain.

Continuity of $D \mathscr{P}^{-1}$ follows from the fact that $C^{\infty}$ satisfies the Heine-Borel property and from the uniqueness of solutions to the WHLS. It follows that $D \mathscr{P}^{-1}$ is a smooth tame map by [4, theorem II.3.1.1]. By the Nash-Moser inverse function theorem $\mathscr{P}$ is locally invertible, and this concludes the proof in the euclidean case.

\subsection{General Case}

The space $\mathbf{E} \subset C^{\infty}([0, T] \times \mathcal{N}, \mathcal{M})$ of time-dependent immersions from $\mathcal{N}$ to the manifold $\mathcal{M}$ is a Fréchet manifold. For $u \in \mathbf{E}$ the operator

$$
\mathfrak{P}(u)=\bar{\nabla}_{\partial_{t}} \partial_{t} u-\frac{d \mu_{t}}{d \hat{\mu}}\left(-H(u)+\frac{\varrho}{\operatorname{Vol}(u)}\right) v
$$

is a vector field along $u$. In order to employ a similar strategy as for the euclidean case, we will in the following translate our problem to an equivalent problem for maps in the Fréchet space $C^{\infty}\left([0, T] \times \mathcal{N}, \mathbb{R}^{d}\right)$.

By the Nash embedding theorem we can suppose that the ambient manifold $\mathcal{M}$ is isometrically embedded into $\mathbb{R}^{d}$ by $\iota: \mathcal{M} \rightarrow \mathbb{R}^{d}$ for some $d$. We will in the following derive an extrinsic form of the Euler-Lagrange equation (EQ) similar to the extrinsic form of wave maps (see, e.g., [9]) and the extrinsic form of the evolution equation for magnetic geodesics [6].

Let $\pi_{\mathcal{M}}$ be the closest point projection to $\iota(\mathcal{M})$ that can be defined on a neighborhood

$$
\tilde{\mathcal{M}}=\left\{x+v\left|x \in \iota(\mathcal{M}), v \in\left(T_{x} \iota(\mathcal{M})\right)^{\perp},\right| v \mid<\delta(x)\right\},
$$


of $\iota(\mathcal{M})$ and is smooth there. Here $\delta$ is a positive smooth function on $\iota(\mathcal{M})$. Now the second fundamental form of $\mathcal{M}$ is given by

$$
\bar{h}_{\alpha \beta}=\bar{\partial}_{\alpha} \bar{\partial}_{\beta} \iota-\bar{\Gamma}_{\alpha \beta}^{\gamma} \bar{\partial}_{\gamma} \iota \text {. }
$$

This is normal to $\iota(\mathcal{M})$ and so $D \pi_{\mathcal{M}}(\iota(p))\left(\bar{h}_{\alpha \beta}\right)=0$ for $p \in \mathcal{M}$. Since $\iota=\pi_{\mathcal{M}} \circ \iota$ we have

$$
\begin{aligned}
\bar{\partial}_{\alpha} \bar{\partial}_{\beta} \iota-\bar{\Gamma}_{\alpha \beta}^{\gamma} \bar{\partial}_{\gamma} \iota & =D_{A} D_{B} \pi_{\mathcal{M}} \bar{\partial}_{\beta} \iota \bar{\partial}_{\alpha} \iota^{B}+D \pi_{\mathcal{M}}\left(\bar{\partial}_{\alpha} \bar{\partial}_{\beta} \iota-\bar{\Gamma}_{\alpha \beta}^{\gamma} \bar{\partial}_{\gamma} \iota\right) \\
& =D_{A} D_{B} \pi_{\mathcal{M}} \bar{\partial}_{\beta} \iota^{A} \bar{\partial}_{\alpha} \iota^{B} .
\end{aligned}
$$

Here $D_{A}$ is the derivative in the direction of the canonical basis vector $e_{A}$ in $\mathbb{R}^{d}$.

Now if $u:[0, T] \times \mathcal{N} \rightarrow \iota(\mathcal{M})$ we can write $u=\iota \circ \widehat{u}$ with $\hat{u}:[0, T] \times \mathcal{N} \rightarrow \mathcal{M}$. We write

$$
\tilde{\square} u=\partial_{t}^{2} u-\frac{d \mu_{t}}{d \hat{\mu}} g^{i j}\left(\partial_{i} \partial_{j} u-\Gamma_{i j}^{k} \partial_{k} u\right)
$$

and compute using (4.2)

$$
\begin{aligned}
& \tilde{\square} u-\frac{d \mu_{t}}{d \hat{\mu}}(u) \frac{\varrho}{\operatorname{Vol}(u)} v(u) \\
&- D_{A} D_{B} \pi_{\mathcal{M}}(u)\left(\partial_{t} u^{A} \partial_{t} u^{B}-\frac{d \mu_{t}}{d \hat{\mu}}(u) g^{i j} \partial_{i} u^{A} \partial_{j} u^{B}\right) \\
& \quad=D \iota\left(\bar{\nabla}_{\partial_{t}} \partial_{t} \hat{u}-\frac{d \mu_{t}}{d \hat{\mu}}(\widehat{u})\left(-H(\widehat{u})+\frac{\varrho}{\operatorname{Vol}(\hat{u})}\right) v(\widehat{u})\right) .
\end{aligned}
$$

Hence $\hat{u}:[0, T] \times \mathcal{N} \rightarrow \mathcal{M}$ solves $(\mathrm{EQ})$ if and only if $u:[0, T] \times \mathcal{N} \rightarrow \iota(\mathcal{N})$ solves

$$
\begin{aligned}
\tilde{\square} u-\frac{d \mu_{t}}{d \hat{\mu}} & (u) \frac{\varrho}{\operatorname{Vol}(u)} v(u) \\
& \quad-D_{A} D_{B} \pi_{\mathcal{M}}(u)\left(\partial_{t} u^{A} \partial_{t} u^{B}-\frac{d \mu_{t}}{d \widehat{\mu}}(u) g^{i j} \partial_{i} u^{A} \partial_{j} u^{B}\right)=0 .
\end{aligned}
$$

We will extend this equation for functions $u:[0, T] \times \mathcal{N} \rightarrow \tilde{\mathcal{M}} \subset \mathbb{R}^{d}$ that do not necessarily map to $\iota(\mathcal{M})$. We will do this in such a way that the linearization is a WHLS in order to apply the Nash-Moser argument for this new equation. Let $\pi_{\Sigma_{t}^{\perp}}(u)$ be the projection onto the normal space of $\Sigma_{t}=u(t, \mathcal{N})$, i.e., $\pi_{\Sigma_{t}^{\perp}}(u) V=$ $V-g^{i j}\left\langle V, \partial_{j} u\right\rangle \partial_{i} u$ for $V \in \mathbb{R}^{d}$. We replace $v(u)$ by $\widetilde{v}(u)=\pi_{\Sigma_{t}^{\perp}}(u) v\left(\pi_{\mathcal{M}} \circ u\right)$, i.e., the projection onto the normal space of $\Sigma_{t}$ of the normal vector $v\left(\pi_{\mathcal{M}} \circ u\right)$ in $\iota(\mathcal{M})$ of the map $\pi_{\mathcal{M}} \circ u:[0, T] \times \mathcal{N} \rightarrow \iota(\mathcal{M})$. If $u$ is close enough in $C^{1}$ to a family of immersions that map to $\iota(\mathcal{M})$, then $\pi_{\mathcal{M}} \circ u$ is also a family of immersions and $v\left(\pi_{\mathcal{M}} \circ u\right)$ is defined. By definition $\widetilde{v}(u)$ is normal to $\partial_{i} u$ and $v\left(\pi_{\mathcal{M}} \circ u\right)$ is an element of $T_{\pi_{\mathcal{M}}(u)^{\iota}}(\mathcal{M})$. We define $\widetilde{\operatorname{Vol}}(u):=\operatorname{Vol}\left(\pi_{\mathcal{M}} \circ u\right)$. We define further $\Pi_{u}\left(\partial_{\alpha} u, \partial_{\beta} u\right)=\pi_{\Sigma_{t}^{\perp}}(u) D_{A} D_{B} \pi_{\mathcal{M}}(u) \partial_{\alpha} u^{A} \partial_{\beta} u^{B}$. 
Then we want to solve the equation

$$
\tilde{\square} u=\frac{d \mu_{t}}{d \widehat{\mu}} \frac{\varrho}{\widetilde{\operatorname{Vol}}(u)} \widetilde{v}+\Pi_{u}\left(\partial_{t} u, \partial_{t} u\right)-\frac{d \mu_{t}}{d \widehat{\mu}} g^{i j} \Pi_{u}\left(\partial_{i} u, \partial_{j} u\right)
$$

subject to given initial conditions.

Assume $u$ solves (4.4). It is clear by definition that if $u$ maps to $\iota(\mathcal{M})$, then we have solved (4.3). We will prove in the following lemma that if $u$ maps to $\iota(\mathcal{M})$ initially and the initial velocity is tangent to $\iota(\mathcal{M})$, then $u$ maps to $\iota(\mathcal{M})$ for all time.

LemMA 4.3. Let $u:[0, T] \times \mathcal{N} \rightarrow \tilde{\mathcal{M}} \subset \mathbb{R}^{d}$ be a smooth solution of equation (4.4) with $u(0, x) \in \iota(\mathcal{M})$ and $\partial_{t} u(0, x) \in T_{u(0, x)} \iota(\mathcal{M})$ for all $x \in \mathcal{N}$. Then $u(t, x) \in$ $\iota(\mathcal{M})$ for all $(t, x) \in[0, T] \times \mathcal{N}$ and $\hat{u}=\iota^{-1} \circ$ u solves $(\mathrm{EQ})$ with $\widehat{u}(0)=\iota^{-1} \circ u(0)$ and $\partial_{t} \widehat{u}(0)=D \iota^{-1}\left(\partial_{t} u(0)\right)$.

Proof. Define $\pi_{\mathcal{M}}^{\perp}(x)=x-\pi_{\mathcal{M}}(x)$. Then clearly $D \pi_{\mathcal{M}}^{\perp}=1-D \pi_{\mathcal{M}}$ and $D_{A} D_{B} \pi_{\mathcal{M}}^{\perp}=-D_{A} D_{B} \pi_{\mathcal{M}}$. We compute

$$
\begin{aligned}
& \partial_{t}^{2}\left(\pi_{\mathcal{M}}^{\perp} \circ u\right)-\frac{d \mu_{t}}{d \hat{\mu}} g^{i j}\left(\partial_{i} \partial_{j}\left(\pi_{\mathcal{M}}^{\perp} \circ u\right)-\Gamma_{i j}^{k} \partial_{k}\left(\pi_{\mathcal{M}}^{\perp} \circ u\right)\right) \\
&=\frac{\varrho}{\widehat{\operatorname{Vol}}(u)} \frac{d \mu_{t}}{d \widehat{\mu}} D_{C} \pi_{\mathcal{M}}^{\perp}(u) v^{C}\left(\pi_{\mathcal{M}} \circ u\right) \\
& \quad-\frac{\varrho}{\widehat{\operatorname{Vol}}(u)} \frac{d \mu_{t}}{d \widehat{\mu}}\left\langle v\left(\pi_{\mathcal{M}} \circ u\right), \partial_{l} u\right\rangle g^{k l} \partial_{k}\left(\pi_{\mathcal{M}}^{\perp} \circ u\right) \\
& \quad-D_{C} \pi_{\mathcal{M}}(u) D_{A} D_{B} \pi_{\mathcal{M}}^{C}(u)\left(\partial_{t} u^{A} \partial_{t} u^{B}-\frac{d \mu_{t}}{d \widehat{\mu}} g^{i j} \partial_{i} u^{A} \partial_{j} u^{B}\right) \\
&-\left\langle D_{A} D_{B} \pi_{\mathcal{M}}(u), \partial_{l} u\right\rangle g^{k l}\left(\partial_{t} u^{A} \partial_{t} u^{B}-\frac{d \mu_{t}}{d \hat{\mu}} g^{i j} \partial_{i} u^{A} \partial_{j} u^{B}\right) \partial_{k}\left(\pi_{\mathcal{M}}^{\perp} \circ u\right) .
\end{aligned}
$$

If $u$ maps to $\iota(\mathcal{M})$, then (4.5) is a linear wave equation for $\pi_{\mathcal{M}}^{\perp} \circ u$ because then we know that $D_{C} \pi_{\mathcal{M}}^{\perp}(u) \nu^{C}\left(\pi_{\mathcal{M}} \circ u\right)=0$ and $D_{C} \pi_{\mathcal{M}}(u) D_{A} D_{B} \pi_{\mathcal{M}}^{C}(u) \partial_{\alpha} u^{A} \partial_{\beta} u^{B}=$ 0 since then $D_{A} D_{B} \pi_{\mathcal{M}}^{C}(u) \partial_{\alpha} u^{A} \partial_{\beta} u^{B}$ is normal to $\iota(\mathcal{M})$. If $u$ does not map to $\iota(\mathcal{M})$, then we can estimate these terms in terms of the distance $\left|\pi \frac{\perp}{\mathcal{M}}(u)\right|$ of $u$ to $\iota(\mathcal{M})$. Hence if we define the energy

$$
e(t)=\frac{1}{2} \int_{\mathcal{N}}\left|\partial_{t}\left(\pi_{\mathcal{M}}^{\perp} \circ u\right)\right|^{2} d \hat{\mu}+\frac{1}{2} \int_{\mathcal{N}}\left|\nabla\left(\pi_{\mathcal{M}}^{\perp} \circ u\right)\right|^{2} d \mu_{t}+\frac{1}{2} \int_{\mathcal{N}}\left|\pi_{\mathcal{M}}^{\perp} \circ u\right|^{2} d \hat{\mu},
$$

it is not hard to check that $\partial_{t} e(t) \leq C e(t)$. Here the constant $C$ may also depend on $u$. Since $e(0)=0$ we conclude by Gronwall's lemma that $e(t)=0$ for all $t \in[0, T]$.

Proof of Theorem 4.1 (General CASE). To conclude the short-time existence proof in the general case, we just note that the linearization of 4.4 can be written as a WHLS. For the bundle $\mathcal{V}$ we take $\mathcal{V}=\mathcal{N} \times \mathbb{R}^{d}$. For $\tau_{k}$ we simply take $\partial_{k} u$ and for $v_{A}$ we take a local frame for the space orthogonal to $\tau_{k}$ with 
$v_{1}=\tilde{v}=\pi_{\Sigma_{t}^{\perp}}(u) v\left(\pi_{\mathcal{M}} \circ u\right)$. We also remark that if $u_{\varepsilon}$ is a variation of $u$ with $\left.\partial_{\varepsilon}\right|_{\varepsilon=0} u_{\varepsilon}=V=\phi_{(\alpha)}^{A} v_{A}^{(\alpha)}+\psi_{(\alpha)}^{k} \tau_{k}^{(\alpha)}$, then

$$
\begin{aligned}
\partial_{\varepsilon} \widetilde{\operatorname{Vol}}(u)= & \int_{\mathcal{N}}\left\langle v\left(\pi_{\mathcal{M}} \circ u\right), V\right\rangle d \mu_{t} \\
= & \sum_{\alpha} \int_{\mathcal{N}} \eta_{\alpha}\left\langle v\left(\pi_{\mathcal{M}} \circ u\right), v_{B}\right\rangle \phi_{(\alpha)}^{B} \frac{d \mu_{t}}{d \hat{\mu}} d \hat{\mu} \\
& +\sum_{\alpha} \int_{\mathcal{N}} \eta_{\alpha}\left\langle v\left(\pi_{\mathcal{M}} \circ u\right), \tau_{l}\right\rangle \psi_{(\alpha)}^{l} \frac{d \mu_{t}}{d \hat{\mu}} d \hat{\mu}
\end{aligned}
$$

where $\eta_{\alpha}$ is a partition-of-unity subordinate to the sets $x_{\alpha}^{-1}\left(B_{2}(0)\right)$. The variation of the volume only occurs in the $v_{1}$-part of the system.

If $V=\psi^{k} \tau_{k}$ is tangential, then only the variation of $d \mu_{t}$ gives spatial derivatives of $V$ since all other terms are diffeomorphism invariant. But this term always accompanies a normal term. This is the reason for defining $\Pi$ and $\widetilde{v}$ with the additional projection to the normal space of $\Sigma_{t}$.

The other conditions are easy to check, and we conclude that the linearization of (4.4) is indeed a WHLS. The Nash-Moser argument applies, and this concludes the proof of Theorem 4.1 in the general case.

\section{Continuation Criterion}

THEOREM 5.1. Let $u:[0, T) \times \mathcal{N} \rightarrow \mathcal{M}$ be a solution of $(\mathrm{EQ})$. Assume that for all $t \in[0, T)$

$$
\|u(t)\|_{C^{4}}+\left\|\partial_{t} u(t)\right\|_{C^{4}} \leq K
$$

for some $K>0$. Then there exists $\delta>0$ such that $u$ can be extended to a solution $\tilde{u}:[0, T+\delta] \times \mathcal{N} \rightarrow \mathcal{M}$ of $(\mathrm{EQ})$.

Remark 5.2. This statement can be formulated as a singularity criterion: If the solution $u$ cannot be extended beyond time $T$, then $\|u\|_{C^{4}}+\left\|\partial_{t} u\right\|_{C^{4}}$ becomes unbounded as $t \rightarrow T$.

Definition 5.3. Let $u:[0, T) \times \mathcal{N} \rightarrow \mathcal{M}$. Define

$$
\begin{aligned}
\sigma & =\left\langle\partial_{t} u, v\right\rangle, & S_{i} & =\left\langle\partial_{t} u, \partial_{i} u\right\rangle, \\
\beta_{i} & =\left\langle\bar{\nabla}_{\partial_{i}} \partial_{t} u, v\right\rangle, & B_{i m} & =\left\langle\bar{\nabla}_{\partial_{i}} \partial_{t} u, \partial_{m} u\right\rangle,
\end{aligned}
$$

where $\bar{\nabla}_{\partial_{i}} \partial_{t} u^{\alpha}=\partial_{i} \partial_{t} u^{\alpha}+\bar{\Gamma}_{\beta \gamma}^{\alpha}(u) \partial_{i} u^{\beta} \partial_{t} u^{\gamma}$. Hence we have

$$
\partial_{t} u=\sigma v+S^{i} \partial_{i} u \quad \text { and } \quad \bar{\nabla}_{\partial_{i}} \partial_{t} u=\beta_{i} v+B_{i}{ }^{k} \partial_{k} u .
$$

Remark 5.4. As in Section 4.2 we assume that $\iota: \mathcal{M} \rightarrow \mathbb{R}^{d}$ is an isometric embedding, and if we identify $\mathcal{M}$ and $\iota(\mathcal{M})$, we can assume $\mathcal{M} \subset \mathbb{R}^{d}$. When we consider norms of $u$, we will take the norm of $u:[0, T) \times \mathcal{N} \rightarrow \mathbb{R}^{d}$ as a map into $\mathbb{R}^{d}$. 
We assume that the metric $\bar{g}$ of the ambient manifold $\mathcal{M}$ and all its derivatives are uniformly bounded in local coordinates. We assume that the second fundamental form of $\mathcal{M}$ and its derivatives are uniformly bounded.

LEMMA 5.5. Let $u$ be a solution of (EQ). Let

$$
\begin{aligned}
\left(\phi^{A}\right)_{A=1, \ldots, 1+n^{2}}= & \left(\sigma,\left(h_{i j}\right)_{i, j=1, \ldots, n}\right), \\
\left(\psi^{k}\right)_{k=1, \ldots, d+n d+n+n^{2}+n^{3}=} & \left(\left(u^{A}\right)_{A=1, \ldots, d},\left(\partial_{i} u^{A}\right)_{\substack{i=1, \ldots, n, A=1, \ldots, d}},\left(S^{j}\right)_{j=1, \ldots, n},\right. \\
& \left.\left(B_{l}{ }^{m}\right)_{l, m=1, \ldots, n},\left(\Gamma_{i j}^{k}-\hat{\Gamma}_{i j}^{k}\right)_{i, j, k=1, \ldots, n}\right),
\end{aligned}
$$

where $\widehat{\Gamma}_{i j}^{k}$ are the Christoffel symbols of a reference metric $g_{0}$ that has $d \hat{\mu}$ as its surface element. Then $\left(\phi^{A}\right)$ and $\left(\psi^{k}\right)$ satisfy

$$
\begin{aligned}
\partial_{t}^{2} \phi^{A}(t, x)-L^{A} \phi(t, x) & =F^{A}\left(x, \phi, D \phi, \partial_{t} \phi, \psi, D \psi, \partial_{t} \psi, \operatorname{Vol}(u)\right), \\
\partial_{t}^{2} \psi^{k} & =G^{k}\left(x, \phi, D \phi, \partial_{t} \phi, \psi, \partial_{t} \psi, \operatorname{Vol}(u)\right),
\end{aligned}
$$

where $F^{A}$ and $G^{k}$ are smooth functions in all their arguments such that if $\|\phi\|_{C^{1}}+$ $\left\|\partial_{t} \phi\right\|_{C^{0}}+\|\psi\|_{C^{1}}+\left\|\partial_{t} \psi\right\|_{C^{0}} \leq K$ and $\operatorname{det}\left(g_{i j}\right)>\lambda_{1}$ for some $K, \lambda_{1}>0$, then $F^{A}$ and $G^{A}$ and all their derivatives stay bounded. The operator $L$ is given by $L \phi=\frac{d \mu_{t}}{d \widehat{\mu}} \Delta \phi$.

PROOF. The proof is a tedious calculation. We just motivate it by the following informal consideration. If $\partial_{\alpha}$ is a derivative, then differentiating our equation $\mathfrak{P}(x, u)=0$ gives

$$
0=D_{u} \mathfrak{P}(x, u)\left\{\partial_{\alpha} u\right\}+D_{x^{\alpha}} \mathfrak{P}(x, u) .
$$

For instance, with $\partial_{\alpha}=\partial_{t}$ we get the evolution equation for $\sigma$ and $S^{i}$. Regarding the structure of the linearized operator $D \mathfrak{P}$ as in (4.1), it is clear that the normal part $\sigma$ satisfies a wave equation and the tangential part $S^{i}$ satisfies an ODE, which means that no spatial derivatives of $S^{i}$ occur in the equation for $S^{i}$.

Differentiating once more using $\partial_{\beta}$, we get

$$
\begin{aligned}
0= & D_{u \mathfrak{P}}(x, u)\left\{\partial_{\beta} \partial_{\alpha} u\right\}+D_{u}^{2} \mathfrak{P}(x, u)\left\{\partial_{\alpha} u, \partial_{\beta} u\right\}+D_{u} D_{x^{\alpha}} \mathfrak{P}(x, u)\left\{\partial_{\beta} u\right\} \\
& +D_{x^{\beta}} D_{u} \mathfrak{P}(x, u)\left\{\partial_{\alpha} u\right\}+D_{x^{\beta}} D_{x^{\alpha}} \mathfrak{P}(x, u) .
\end{aligned}
$$

Taking $\partial_{\beta} \partial_{\alpha}=\bar{\nabla}_{\partial_{i}} \partial_{j}$ and decomposing $\bar{\nabla}_{\partial_{i}} \partial_{j} u=-h_{i j} v+\Gamma_{i j}^{k} \partial_{k} u$, we obtain a wave equation for $h_{i j}$ and an ODE for $\Gamma_{i j}^{k}$. With $\bar{\nabla}_{\partial_{t}} \partial_{i}$ we obtain the evolution equations for $\beta_{i}$ and $B_{i}{ }^{k}$. Since we can always express

$$
\beta_{i}=\left\langle\bar{\nabla}_{\partial_{i}}\left(\sigma v+S^{k} \partial_{k} u\right), v\right\rangle=\partial_{i} \sigma-S^{k} h_{i k},
$$

we can eliminate all occurrences of $\beta_{i}$ in the other equations and do not need the evolution equation for $\beta_{i}$. Since $\mathfrak{P}$ is a second-order operator no more than third derivatives will occur due to the decomposition into normal and tangential parts. Third derivatives can always be expressed in terms of at most first derivatives of 
the quantities $D u, \sigma, S^{i}, h_{i j}, \Gamma_{i j}^{k}$, and $B_{i}{ }^{k}$. The claimed structure of the system is apparent since the leading-order term is always the linearized operator.

Note that due to the assumption $\operatorname{det}\left(g_{i j}\right)>\lambda_{1}$, terms such as $g^{i j}$ and $v$ will not blow up and $\operatorname{Vol}(u)^{-1}$ is bounded in view of the energy conservation.

Lemma 5.6. Let $u:[0, T) \times \mathcal{N} \rightarrow \mathcal{M}$ with $\left\|\partial_{t} u\right\|_{C^{1}}+\|u\|_{C^{1}} \leq K$ for some $K>$ 0 . Then the metrics $g_{i j}(t)$ for all different times are equivalent, and they converge as $t \rightarrow T$ uniformly to a positive definite metric $g_{i j}(T)$, which is continuous and also equivalent.

PROOF. This follows from [5, lemma 14.2] using the assumed bound on

$$
\partial_{t} g_{i j}=\left\langle\bar{\nabla}_{\partial_{t}} \partial_{i} u, \partial_{j} u\right\rangle+\left\langle\partial_{i} u, \bar{\nabla}_{\partial_{t}} \partial_{j} u\right\rangle .
$$

PRoOF OF THEOREM 5.1. Let $s \geq\left\lfloor\frac{n}{2}\right\rfloor+2$.

(1) Estimating the Operator $L$. The coefficients of $L=\frac{d \mu_{t}}{d \hat{\mu}} \Delta$ contain $g^{i j}$, Christoffel symbols, and derivatives of Christoffel symbols. Lemma 5.6 implies $\operatorname{det}\left(g_{i j}\right) \geq \lambda_{1}$ for some $\lambda_{1}>0$. By the Moser inequalities we can estimate

$$
[L]_{s} \leq C\left(1+\|u\|_{s+3}\right)
$$

with $C$ depending on $K$ since $L$ contains no more than third derivatives of $u$. Clearly, also by the assumption, $[L]_{C^{1}} \leq C_{K}$. Similarly, $\left[\partial_{t} L\right]_{s} \leq C\left(1+\|u\|_{s+3}+\right.$ $\left.\left\|\partial_{t} u\right\|_{s+3}\right)$ and $\left[\partial_{t} L\right]_{C^{1}} \leq C_{K}$.

(2) Application of the Estimate FOR WHLS. In order to apply Proposition 3.4 we have to specify the vector bundle $\mathcal{V}$ and the basis in which the system from Lemma 5.5 is a WHLS if we consider the right-hand side $F^{A}(\cdots), G^{k}(\cdots)$ as fixed functions. For the bundle we take

$$
\begin{aligned}
\mathcal{V}= & (\mathcal{N} \times \mathbb{R}) \oplus\left(T^{*} \mathcal{N} \otimes T^{*} \mathcal{N}\right) \oplus\left(\mathcal{N} \times \mathbb{R}^{d}\right) \oplus\left(\left(\mathcal{N} \times \mathbb{R}^{d}\right) \otimes T^{*} \mathcal{N}\right) \\
& \oplus T \mathcal{N} \oplus\left(T^{*} \mathcal{N} \otimes T \mathcal{N}\right) \oplus\left(T^{*} \mathcal{N} \otimes T^{*} \mathcal{N} \otimes T \mathcal{N}\right) .
\end{aligned}
$$

Let $\left(x_{\alpha}, U_{\alpha}\right)$ be a local coordinate chart with canonical tangent vectors $\partial_{k}$ and $d x^{i}$ its dual covectors. For $v_{A}$ we take

$$
\left\{v_{A}\right\}_{A=1, \ldots, 1+n^{2}}=\left\{(1,0,0,0,0,0,0),\left(0, d x^{i} \otimes d x^{j}, 0,0,0,0,0\right)_{i, j=1, \ldots, n}\right\},
$$

and for $\tau_{k}$ we take

$$
\begin{aligned}
\left\{\tau_{k}\right\}_{k=1, \ldots, d+n d+n+n^{2}+n^{3}=\{} & \left(0,0, e_{A}, 0,0,0,0\right)_{A=1, \ldots, d}, \\
& \left(0,0,0, e_{A} \otimes d x^{i}, 0,0,0\right)_{i=1, \ldots, n,}, \\
A=1, \ldots, d & \\
& \left(0,0,0,0, \partial_{k}, 0,0\right)_{k=1, \ldots, n}, \\
& \left(0,0,0,0,0, d x^{i} \otimes \partial_{k}, 0\right)_{i, k=1, \ldots, n}, \\
& \left.\left(0,0,0,0,0,0, d x^{i} \otimes d x^{j} \otimes \partial_{k}\right)_{i, j, k=1, \ldots, n}\right\} .
\end{aligned}
$$


The system for $\phi, \psi$ from Lemma 5.5 is a WHLS with $M=N=P=Q_{1}=$ $Q_{2}=0$ and right-hand side $F^{A}, G^{k}$. By assumption and Lemma 5.5 the righthand side is bounded. It is easy to check using the definitions of $\phi$ and $\psi$ and the assumptions that

$$
\|\phi\|_{C^{2}}+\left\|\partial_{t} \phi\right\|_{C^{2}}+\|\psi\|_{C^{1}}+\left\|\partial_{t} \psi\right\|_{C^{1}} \leq C_{K}
$$

As in (3.2), define

$$
E_{s}(t)=\left\|\partial_{t}^{2} \phi(t)\right\|_{s}+\left\|\partial_{t} \phi(t)\right\|_{s+1}+\|\phi(t)\|_{s+1}+\left\|\partial_{t} \psi(t)\right\|_{s+1}+\|\psi(t)\|_{s+1} .
$$

Hence we can apply Proposition 3.4 to obtain

$$
\begin{aligned}
E_{S}(t) \leq C e^{C t}\left(1+E_{S}(0)+\int_{0}^{t}\right. & \|F(\cdots)\|_{s}+\left\|\partial_{t}(F(\cdots))\right\|_{s} \\
& \left.+\|G(\cdots)\|_{s+1}+[L]_{s}+\left[\partial_{t} L\right]_{s} d t^{\prime}\right) .
\end{aligned}
$$

(3) Estimating the Right-Hand Side. We have to estimate $\|F(\cdots)\|_{s}$, $\left\|\partial_{t}(F(\cdots))\right\|_{s}$, and $\|G(\cdots)\|_{s+1}$. By assumption

$$
|\operatorname{Vol}(u)|=\left|\operatorname{Vol}_{0}+\int_{0}^{t} \int_{\mathcal{N}}\left\langle\partial_{t} u, v\right\rangle d \mu_{t} d t^{\prime}\right| \leq C
$$

and

$$
\left|\partial_{t} \operatorname{Vol}(u)\right|=\left|\int_{\mathcal{N}}\left\langle\partial_{t} u, v\right\rangle d \mu_{t}\right| \leq C .
$$

By assumption and from Lemma 5.5, we can apply the third Moser inequality [11, chap. 13, prop. 3.9],

$$
\|F(\cdots)\|_{s}+\|G(\cdots)\|_{s+1}+\left\|\partial_{t}(F(\cdots))\right\|_{s} \leq C\left(1+E_{s}+\|\phi\|_{s+2}+\left\|\partial_{t}^{2} \psi\right\|_{s}\right) .
$$

Using the equation for $\partial_{t}^{2} \psi$, we can estimate

$$
\left\|\partial_{t}^{2} \psi\right\|_{s} \leq C\|G(\cdots)\|_{s} \leq C\left(1+E_{s}+\|\phi\|_{s+2}\right)
$$

We use the elliptic estimate and the equation (similarly as in (3.8)) to estimate

$$
\begin{aligned}
\|\phi\|_{s+2} & \leq C\left(\|L \phi\|_{s}+\|\phi\|_{s+1}+[L]_{s}\right) \\
& \leq C\left(\left\|\partial_{t}^{2} \phi\right\|_{s}+\|F(\cdots)\|_{s}+\|\phi\|_{s+1}+\|u\|_{s+3}+1\right) \\
& \leq C\left(1+E_{s}\right) .
\end{aligned}
$$

The initial energy $E_{S}(0)$ is fixed. So we obtain the estimate

$$
E_{S}(t) \leq C_{T, K}\left(1+\int_{0}^{t} 1+E_{S}\left(t^{\prime}\right) d t^{\prime}\right)
$$

We use Gronwall's lemma to conclude

$$
\|\phi(t)\|_{s+1}+\left\|\partial_{t} \phi(t)\right\|_{s+1}+\left\|\partial_{t}^{2} \phi(t)\right\|_{s}+\|\psi(t)\|_{s+1}+\left\|\partial_{t} \psi(t)\right\|_{s+1} \leq C
$$


for all $0 \leq t<T$. This can be done for any $s \geq\left\lfloor\frac{n}{2}\right\rfloor+2$. This implies that all derivatives of $u$ are bounded uniformly in $t$.

It is then easy to see that $u(t)$ and $\partial_{t} u(t)$ converge in $C^{\infty}$ to a smooth immersion $u(T)$ and corresponding velocity $\partial_{t} u(T)$. Then we can apply the short-time existence result Theorem 4.1 to extend the solution.

\section{Stability Estimates}

Assume that we have a solution $\tilde{u}:[0, \tilde{T}) \times \mathcal{N} \rightarrow \mathcal{M}$ of $(\mathrm{EQ})$. For instance, this could be one of the special solutions in Section 2.3 with $\widetilde{T}=\infty$. All quantities with ${ }^{\sim}$ will refer to $\tilde{u}$, e.g., $\tilde{h}_{i j}$ is the second fundamental form of $\tilde{u}$. We intend to prove the following theorem:

TheOrem 6.1. Let $s \geq\left\lfloor\frac{n}{2}\right\rfloor+2, \widetilde{T} \in \mathbb{R}^{+} \cup\{\infty\}$, and $\tilde{u}:[0, \widetilde{T}) \times \mathcal{N} \rightarrow \mathcal{M}$ be a solution of $(\mathrm{EQ})$. Assume for all $t \in[0, \widetilde{T})$

$$
\|\widetilde{u}(t)\|_{s+4}+\left\|\partial_{t} \tilde{u}(t)\right\|_{s+3} \leq K, \quad \operatorname{det}\left(\widetilde{g}_{i j}\right) \geq \lambda_{1}, \quad \text { and } \quad \widetilde{g}^{i j} \geq \lambda
$$

for some constants $K, \lambda_{1}, \lambda>0$. There exist constants $c_{1}, c_{2}$, and $\varepsilon_{0}>0$ such that if $u_{0}: \mathcal{N} \rightarrow \mathcal{M}$ is an immersion and $u_{1}: \mathcal{N} \rightarrow u_{0}^{*} T \mathcal{N}$ is a vector field along $u_{0}$ with

$$
\left\|u_{0}-\tilde{u}(0)\right\|_{s+4}+\left\|u_{1}-\partial_{t} \tilde{u}(0)\right\|_{s+3} \leq \varepsilon
$$

for some $0<\varepsilon \leq \varepsilon_{0}$, then there exists $T \geq \min \left\{\tilde{T}, c_{1} \log \left(\frac{c_{2}}{\varepsilon}\right)\right\}$ and $u:[0, T] \times$ $\mathcal{N} \rightarrow \mathcal{M}$ that solves

$$
\left\{\begin{array}{l}
\bar{\nabla}_{\partial_{t}} \partial_{t} u=\frac{d \mu_{t}}{d \widehat{\mu}}\left(-H(u)+\frac{\varrho}{\operatorname{Vol}(u)}\right) v \text { for all } t \in[0, T], \\
u(0)=u_{0} \\
\partial_{t} u(0)=u_{1} .
\end{array}\right.
$$

For all $t \in[0, T]$ we have the estimate

$$
\|u(t)-\tilde{u}(t)\|_{s+4}+\left\|\partial_{t} u(t)-\partial_{t} \tilde{u}(t)\right\|_{s+3} \leq C e^{C t} \varepsilon
$$

with $C$ depending on $s$ and $\tilde{u}$.

Corollary 6.2 (Uniqueness). Let $u:[0, T] \times \mathcal{N} \rightarrow \mathcal{M}$ and $\tilde{u}:[0, T] \times \mathcal{N} \rightarrow \mathcal{M}$ be solutions of $(\mathrm{EQ})$ with $u(0)=\tilde{u}(0)$ and $\partial_{t} u(0)=\partial_{t} \tilde{u}(0)$. Then $u(t)=\tilde{u}(t)$ for all $t \in[0, T]$.

LEMMA 6.3. Let $u$ be a solution of $(\mathrm{EQ})$ and $\tilde{u}$ as in Theorem 6.1 Let

$$
\begin{aligned}
\left(\phi^{A}\right)_{A=1, \ldots, 1+n^{2}=} & \left((\sigma-\widetilde{\sigma}),\left(h_{i j}-\tilde{h}_{i j}\right)_{i, j=1, \ldots, n}\right) \\
\left(\psi^{k}\right)_{k=1, \ldots, d+n d+n+n^{2}+n^{3}=} & \left(u^{A}-\widetilde{u}^{A}\right)_{A=1, \ldots, d},\left(\partial_{i} u^{A}-\partial_{i} \widetilde{u}^{A}\right)_{\substack{i=1, \ldots, n \\
A=1, \ldots, d}}, \\
& \left(S^{j}-\widetilde{S}^{j}\right)_{j=1, \ldots, n},\left(B_{l}{ }^{m}-\widetilde{B}_{l}{ }^{m}\right)_{l, m=1, \ldots, n}, \\
& \left.\left(\Gamma_{i j}^{k}-\widetilde{\Gamma}_{i j}^{k}\right)_{i, j, k=1, \ldots, n}\right) .
\end{aligned}
$$


Then $\left(\phi^{A}\right)$ and $\left(\psi^{k}\right)$ satisfy

$$
\begin{aligned}
\partial_{t}^{2} \phi^{A}(t, x)-L^{A} \phi(t, x) & =F^{A}\left(x, \phi, D \phi, \partial_{t} \phi, \psi, D \psi, \partial_{t} \psi, \operatorname{Vol}(u)-\operatorname{Vol}(\tilde{u})\right), \\
\partial_{t}^{2} \psi^{k} & =G^{k}\left(x, \phi, D \phi, \partial_{t} \phi, \psi, \partial_{t} \psi, \operatorname{Vol}(u)-\operatorname{Vol}(\widetilde{u})\right)
\end{aligned}
$$

where $F^{A}$ and $G^{k}$ are smooth functions in all their arguments such that if $\|\phi\|_{C^{1}}+$ $\left\|\partial_{t} \phi\right\|_{C^{0}}+\|\psi\|_{C^{1}}+\left\|\partial_{t} \psi\right\|_{C^{0}} \leq K$ and $\operatorname{det}\left(g_{i j}\right)>\lambda_{1}$, then $F^{A}$ and $G^{A}$ and all its derivatives stay bounded. Furthermore, $F(x, 0)=0$ and $G(x, 0)=0$ and the operator $L$ is given by $L \phi=\frac{d \mu_{t}}{d \widehat{\mu}} \Delta_{g(t)} \phi$.

PROOF. Simply subtract the evolution equations for all the quantities corresponding to $\tilde{u}$ from the evolution equations for the quantities corresponding to $u$. Replace everywhere, e.g., $\sigma=\widetilde{\sigma}+(\sigma-\widetilde{\sigma})$, to write the equations as equations for the differences. Then the claimed structure follows as in the proof of Lemma 5.5 ,

Proof of Theorem 6.1. As in 3.2, define

$$
\begin{aligned}
E_{S}(t)= & \left\|\partial_{t}^{2} \phi(t, \cdot)\right\|_{s}+\left\|\partial_{t} \phi(t, \cdot)\right\|_{s+1}+\|\phi(t, \cdot)\|_{s+1} \\
& +\left\|\partial_{t} \psi(t, \cdot)\right\|_{s+1}+\|\psi(t, \cdot)\|_{s+1}
\end{aligned}
$$

with $\phi$ and $\psi$ from Lemma 6.3. Define

$$
\widetilde{E}_{s}(t)=E_{S}(t)+|\operatorname{Vol}(u(t))-\operatorname{Vol}(\tilde{u}(t))| .
$$

Choose a constant $\kappa>0$ such that $g^{i j} \geq \lambda_{2}>0$ and $\operatorname{det}\left(g_{i j}\right) \geq \lambda_{3}>0$ if $\widetilde{E}_{S}(t) \leq \kappa$. Assume that $\widetilde{E}_{S}(t) \leq \kappa$. We will prove under this assumption that $\widetilde{E}_{S}(t) \leq \frac{1}{2} \kappa$ as long as $t \leq \min \left\{\widetilde{T}, c_{1} \log \left(\frac{c_{2}}{\varepsilon}\right)\right\}$.

Using the Sobolev embedding theorem, we see that the assumptions of Proposition 3.4 are satisfied. With the modifications of Remark 3.5, we obtain

$$
\begin{aligned}
E_{S}(t) \leq C e^{C t} E_{S}(0) & \\
& +C \int_{0}^{t} e^{C\left(t-t^{\prime}\right)}\left(\|F(\cdots)\|_{s}+\left\|\partial_{t}(F(\cdots))\right\|_{s}\right. \\
& +\|G(\cdots)\|_{s+1}+[L]_{s}\|\phi\|_{C^{2}}+\left[\partial_{t} L\right]_{s}\|\phi\|_{C^{2}} \\
& \left.+[L]_{s}\left\|\partial_{t} \phi\right\|_{C^{2}}\right) d t^{\prime} .
\end{aligned}
$$

We carry out the estimates of the right-hand side using the third Moser inequality, similarly to the proof of Theorem 6.1. Since $F(x, 0)=0$ and $G(x, 0)=0$, we do not need the constant term on the right-hand side

$$
\begin{aligned}
& \|F(\cdots)\|_{s}+\left\|\partial_{t}(F(\cdots))\right\|_{s}+\|G(\cdots)\|_{s+1} \leq \\
& C_{K}\left(E_{s}+\|\operatorname{Vol}(u)-\operatorname{Vol}(\widetilde{u})\|_{0}+\left\|\partial_{t} \operatorname{Vol}(u)-\partial_{t} \operatorname{Vol}(\widetilde{u})\right\|_{0}\right) .
\end{aligned}
$$


We also have

$$
[L]_{S}\|\phi\|_{C^{2}}+\left[\partial_{t} L\right]_{S}\|\phi\|_{C^{2}}+[L]_{S}\left\|\partial_{t} \phi\right\|_{C^{2}} \leq C_{\kappa}\left(1+E_{S}\right) E_{s} \leq C_{\kappa} E_{s} .
$$

Now $\partial_{t} \operatorname{Vol}(u)=\int_{\mathcal{N}}\left\langle\partial_{t} u, v\right\rangle d \mu_{t}$ and hence

$$
\left|\partial_{t} \operatorname{Vol}(u)-\partial_{t} \operatorname{Vol}(\tilde{u})\right| \leq C_{\kappa} E_{s} .
$$

We use the fundamental theorem of calculus to estimate

$$
|\operatorname{Vol}(u)-\operatorname{Vol}(\widetilde{u})| \leq C e^{C t}\left|\operatorname{Vol}\left(u_{0}\right)-\operatorname{Vol}(\widetilde{u}(0))\right|+C \int_{0}^{t} e^{C\left(t-t^{\prime}\right)} E_{S}\left(t^{\prime}\right) d t^{\prime} .
$$

Adding 6.1 and 6.2, we get

$$
\widetilde{E}_{S}(t) \leq C e^{C t} \widetilde{E}_{s}(0)+C \int_{0}^{t} e^{C\left(t-t^{\prime}\right)} \widetilde{E}_{S}\left(t^{\prime}\right) d t^{\prime} .
$$

We apply Gronwall's inequality to see that

$$
\widetilde{E}_{S}(t) \leq C e^{C t} \varepsilon \text {. }
$$

Hence if $t \leq C^{-1} \log \left(\frac{\kappa}{2 C \varepsilon}\right)$, then $\widetilde{E}_{S}(t) \leq \frac{1}{2} \kappa$. Define $c_{1}=C^{-1}$ and $c_{2}=\frac{\kappa}{2 C}$. We need $t>0$ and hence $\varepsilon<c_{2}$.

If we choose $\varepsilon_{0}$ such that $\varepsilon_{0}<c_{2}$ and such that $\widetilde{E}_{S}(0) \leq \kappa$, then the bootstrap principle (e.g., [10, prop. 1.21]) shows that our solutions exist and 6.3 holds as long as $t \leq \min \left\{\tilde{T}, c_{1} \log \left(\frac{c_{2}}{\varepsilon}\right)\right\}$.

If we include the difference of the ambient metric to the euclidean metric, we obtain a similar stability result. The proof is similar to that of Theorem 6.1 and is omitted.

THEOREM 6.4. Let $\mathcal{M}=\left(\mathbb{R}^{n+1}, \bar{g}\right)$ and let $\tilde{\mathcal{M}}=\left(\mathbb{R}^{n+1}, \delta\right)$ with the euclidean metric $\delta$ on $\mathbb{R}^{n+1}$. Let $s \geq\left\lfloor\frac{n}{2}\right\rfloor+2$ and $\tilde{u}:[0, \infty) \times \mathcal{N} \rightarrow \tilde{\mathcal{M}}$ be a solution of (EQ), and let for all $t \in[0, \infty)$

$$
\|\tilde{u}(t)\|_{s+4}+\left\|\partial_{t} \tilde{u}(t)\right\|_{s+3} \leq K \text { and } \operatorname{det}\left(\widetilde{g}_{i j}\right) \geq \lambda_{1} \text { and } \widetilde{g}^{i j} \geq \lambda
$$

for some constants $K, \lambda_{1}, \lambda>0$. There exist constants $c_{1}, c_{2}$, and $\varepsilon_{0}, \varepsilon_{1}>0$ such that if $u_{0}: \mathcal{N} \rightarrow \mathcal{M}$ is an immersion and $u_{1}: \mathcal{N} \rightarrow u_{0}^{*} T \mathcal{M}$ is a vector field along $u_{0}$ with

$$
\left\|u_{0}-\tilde{u}(0)\right\|_{s+4}+\left\|u_{1}-\partial_{t} \tilde{u}(0)\right\|_{s+3} \leq \varepsilon
$$

and

$$
\|\bar{g}-\delta\|_{C^{s+4}} \leq \varepsilon^{\prime}
$$

for some $0<\varepsilon \leq \varepsilon_{0}$ and $0<\varepsilon^{\prime} \leq \varepsilon_{1}$, then there exists $T \geq c_{1} \log \left(\frac{c_{2}}{\varepsilon+\varepsilon^{\prime}}\right)$ and $u:[0, T] \times \mathcal{N} \rightarrow \mathcal{M}$ that solves

$$
\left\{\begin{array}{l}
\bar{\nabla}_{\partial_{t}} \partial_{t} u=\frac{d \mu_{t}}{d \widehat{\mu}}\left(-H(u)+\frac{\varrho}{\operatorname{Vol}(u)}\right) v \text { for all } t \in[0, T] \\
u(0)=u_{0} \\
\partial_{t} u(0)=u_{1}
\end{array}\right.
$$


For all $t \in[0, T]$ we have the estimate

$$
\|u(t)-\tilde{u}(t)\|_{s+4}+\left\|\partial_{t} u(t)-\partial_{t} \tilde{u}(t)\right\|_{s+3} \leq C e^{C t}\left(\varepsilon+\varepsilon^{\prime}\right)
$$

with $C$ depending on $s$ and $\tilde{u}$.

\section{Bibliography}

[1] Barbosa, J. L.; do Carmo, M.; Eschenburg, J. Stability of hypersurfaces of constant mean curvature in Riemannian manifolds. Math. Z. 197 (1988), no. 1, 123-138.

[2] DeTurck, D. M. Deforming metrics in the direction of their Ricci tensors (improved version). Collected papers on Ricci flow. Edited by H. D. Cao, B. Chow, S. C. Chu, and S. T. Yau. Series in Geometry and Topology, 37. International Press, Somerville, Mass., 2003.

[3] Evans, L. C. Partial differential equations. Graduate Studies in Mathematics, 19. American Mathematical Society, Providence, R.I., 1998.

[4] Hamilton, R. S. The inverse function theorem of Nash and Moser. Bull. Amer. Math. Soc. (N.S.) 7 (1982), no. 1, 65-222.

[5] Hamilton, R. S. Three-manifolds with positive Ricci curvature. J. Differential Geom. 17 (1982), no. 2, 255-306.

[6] Koh, D. On the evolution equation for magnetic geodesics. Calc. Var. Partial Differential Equations 36 (2009), no. 3, 453-480.

[7] LeFloch, P. G.; Smoczyk, K. The hyperbolic mean curvature flow. J. Math. Pures Appl. (9) 90 (2008), no. 6, 591-614.

[8] Notz, T. Closed hypersurfaces driven by their mean curvature and inner pressure. Doctoral thesis, Freie Universität Berlin, 2010. Available at: http://www.diss.fuberlin.de/diss/receive/FUDISS_thesis_000000018696.

[9] Shatah, J.; Struwe, M. Geometric wave equations. Courant Lecture Notes in Mathematics, 2. New York University, Courant Institute of Mathematical Sciences, New York; American Mathematical Society, Providence, R.I., 1998.

[10] Tao, T. Nonlinear dispersive equations. Local and global analysis. CBMS Regional Conference Series in Mathematics, 106. Published for the Conference Board of the Mathematical Sciences, Washington, D.C.; by the American Mathematical Society, Providence, R.I., 2006.

[11] Taylor, M. E. Partial differential equations. III. Applied Mathematical Sciences, 117. Springer, New York, 1997.

\section{THILO NOTZ}

Max Planck Institute for Gravitational

Physics (Albert Einstein Institute)

Am Mühlenberg 1

14476 Golm

GERMANY

E-mail: thilo.notz@ymail.com

Received June 2011. 